\title{
Optically Efficient Nonlinear Signal Processing
}

\author{
Alan E. Willner, Fellow, IEEE, Omer Faruk Yilmaz, Student Member, IEEE, Jian Wang, Student Member, IEEE, \\ Xiaoxia Wu, Student Member, IEEE, Antonella Bogoni, Lin Zhang, and Scott R. Nuccio
}

(Invited Paper)

\begin{abstract}
Optical signal processing techniques employ a wide range of devices and various nonlinearities to achieve multiple network functionalities. The choice of nonlinearity can also impact the relative efficiency, both in terms of energy and material consumption, of the signal processing function being implemented. Techniques for some of the important functionalities, wavelength multicasting, wavelength-division multiplexing to time-division multiplexing, add-drop multiplexing, and wavelength exchange are compared in terms of the used optical spectrum, number of pumps required, and optical energy consumed. These include varieties of four-wave mixing, cross-phase modulation, Kerr-effectbased polarization rotation in optical fibers, and three-wave mixing in lithium niobate waveguides (WGs). Future possibilities of greener optical signal processing using on-chip WG technologies are discussed within the scope of recent developments in the dispersion tailored, highly nonlinear WGs.
\end{abstract}

Index Terms-Add-drop multiplexing, multicasting, multiplexing, nonlinear optics, optical fiber communications, optical signal processing, silicon waveguides, wavelength exchange.

\section{INTRODUCTION}

$\mathbf{T}$ ODAY'S networks are growing at incredible rates, driven by both an increase in the number of connections as well as the demand for higher bandwidth applications, mainly video content. This growth places increasingly costly requirements on available resources, including power and raw materials. The ability to feed the growth may eventually become a limiting barrier, driving up the cost of network operation. It has been estimated that by 2030 , the power that will be demanded by the optical communications infrastructure in Japan will be higher than the total energy production of the country [1]. To offset this growing trend, research efforts have focused on the ways to improve the efficiency of these networks, often by leveraging photonic alternatives to provide improved performance with lower material and energy costs [2].

Manuscript received April 8, 2010; revised May 18, 2010; accepted June 8, 2010. Date of publication August 11, 2010; date of current version April 6, 2011. This work was supported in part by the Defense Advanced Research Project Agency under Grant FA8650-08-1-7820.

A. E. Willner, O. F. Yilmaz, J. Wang, X. Wu, L. Zhang, and S. R. Nuccio are with the Ming Hsieh Department of Electrical Engineering, University of Southern California, Los Angeles, CA 90089 USA (e-mail: willner@usc.edu).

A. Bogoni is with the Ming Hsieh Department of Electrical Engineering, University of Southern California, Los Angeles, CA 90089 USA, and also with the Consorzio Nazionale Interuniversitario per le Telecomunicazioni, Pisa 56124, Italy.

Color versions of one or more of the figures in this paper are available online at http://ieeexplore.ieee.org.

Digital Object Identifier 10.1109/JSTQE.2010.2055551
This concept of "green photonics" is driven by several beneficial properties of optics. By taking advantage of the relatively unlimited bandwidth of optics, and the femtosecond response times of photonic materials, green photonic solutions can support single-channel data rates well beyond $100 \mathrm{~Gb} / \mathrm{s}$ in a single element [3], [4]. Since optics does not need to "touch" or operate on each individual bit, a single photonic element has the ability to transparently process a data channel regardless of its data rate or the modulation format of the data that it carries allowing for efficient scaling of network resources [5]-[9]. Similarly, a single photonic element can operate on multiple data channels simultaneously, greatly reducing the need for large fan-outs and redundant parallel processing structures. This may be especially true, as spectral efficiency requirements continue to move networks toward multibit-per-symbol formats that may require extensive parallel processing [10]-[13]. The possibility of elimination of an optical-electrical-optical conversion process with an energy consumption of $\sim 0.5 \mathrm{~nJ} / \mathrm{bit}$ [14] by optical signal processing methods may be advantageous considering the capabilities of operation at line rates $>100 \mathrm{~Gb} / \mathrm{s}$. Furthermore, as optical technologies improve and integrated solutions become increasingly available, optics offers the potential for a continued decrease in the cost per bit over what is currently achievable.

There exists a wide variety of photonic materials capable of providing green operation through optical signal processing, including highly nonlinear specialty fibers, periodically poled lithium niobate (PPLN) waveguides, chalcogenide glass chips, silicon waveguides, and many others. Fiber-based solutions have the advantage of being directly integrated with existing fiber networks and utilizing cheap fiber components for their implementations. Silica-based highly nonlinear fiber (HNLF) is the most common choice, although many structures and materials including photonic crystal fiber, bismuth-oxide-doped fiber (Bi-HNLF), and chalcogenide fibers have shown great potential in miscellaneous optical signal processing applications, such as wavelength conversion, regeneration, and format conversion [15]-[20].

While direct integration of fiber may have some limitations, both silicon $(\mathrm{Si})$ and chalcogenide-based alternatives have the potential for direct chip-level integration. Leveraging the mature Si processing industry, Si photonics has become one of the driving goals of current green photonics research. With new low-loss processes, impressive results show the potential of waveguide (WG) devices in ultrahigh-speed optical signal processing up to $1.28 \mathrm{~Tb} / \mathrm{s}$ [8], [9].

Many of these materials utilize a variety of both $\chi^{(2)}$ : $\chi^{(2)}$ and $\chi^{(3)}$ nonlinear interactions, including cascaded 
second-harmonic generation and difference-frequency generation (cSHG/DFG), and cascaded sum- and difference-frequency generation (cSFG/DFG) in PPLN waveguides, self-phase modulation, cross-phase modulation(XPM), and degenerate and nondegenerate four-wave mixing (FWM) in fibers, chalcogenide chips, and silicon WGs. The choice of nonlinear interaction can be critical for implementing a green photonic function in the most efficient matter.

The total energy consumed is often estimated by the number of high-power optical lasers required to perform the signal processing function. Coupling and filtering losses, and the addition of high-power optical amplifiers limited with the pump-laser efficiencies often greatly increase the power energy consumption, and number of components necessary. By utilizing nonlinear processes that require a minimum number of high-power pump lasers, it is possible to demonstrate the large potential of green photonics. To this end, each optical technique is compared using the total optical energy per bit required to achieve the desired function. In this manner, wide variation in equipment and experimental setups used can be minimized, e.g., an amplified and filtered pump laser can be exchanged with a single standing high-power laser unit eliminating a high-power amplifier and a filter, and a more direct comparison between different functions, devices, and nonlinearities is possible.

In this paper, we investigate different optical signal processing techniques that employ a wide range of devices and various nonlinearities to achieve network functionalities. While a wide range of functions can be envisioned for future optical systems, four network functionalities have been chosen as examples due to their diverse use of devices and the varying nonlinearities employed. We will focus on the energy-efficient methods to enable several optical signal processing applications, including wavelength multicasting, wavelength-division multiplexing (WDM) to optical time-division multiplexing (OTDM) conversion, optical add-drop multiplexing, and wavelength exchange. The performances of different techniques are studied from an optical energy consumption view (energy/bit). Optical energy per bit for these nonlinear processes often scales inversely with data rate. In Section II, we give a brief introduction of the basic concept of multicasting, multiplexing, and wavelength exchange. In Section III, we describe potential green methods to realize these optical signal processing applications, addressing several issues such as resource savings and optical energy consumption. Nonlinear integrated WGs, potential candidates for efficient optical signal processing, are discussed in Section IV. Finally, a brief summary is given in Section $\mathrm{V}$.

\section{CONCEPT}

Recent experimental demonstrations are presented, covering wavelength multicasting, WDM-to-TDM multiplexing, add/drop multiplexing, and wavelength exchange. In this section, we describe the operating principle of each application.

\section{A. Wavelength Multicasting}

Wavelength multicasting is the selective distribution of data to predetermined wavelengths [21], [22]. The input signal at a

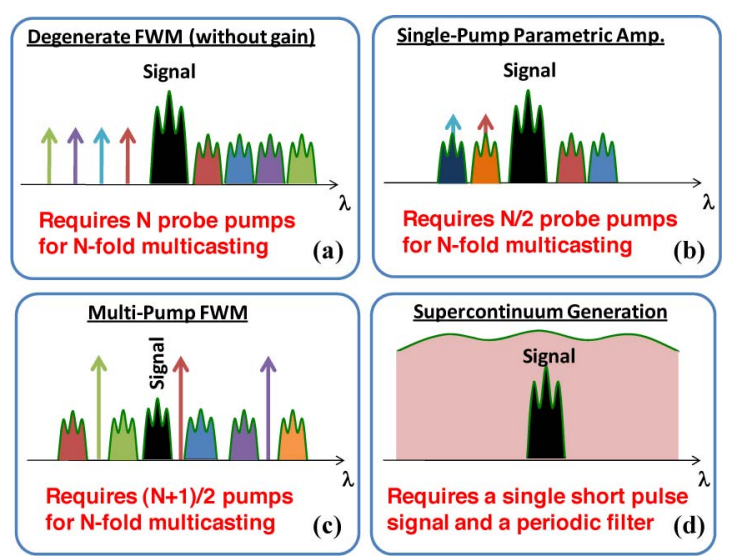

Fig. 1. Conceptual spectra for various method of multicasting. (a) FWM. (b) Parametric amplification. (c) Multiple pump FWM. (d) Supercontinuumbased multicasting schemes.

given wavelength is copied to multiple output wavelengths using different optical signal processing methods. These methods differ in the number of pumps and seed lasers depending on the optical nonlinearity being used to generate the new output signals. A potentially important characteristic of an all-optical multicaster is the minimal use of additional pump lasers that consume added energy and spectrum.

Conceptual spectra for different methods of multicasting are shown in Fig. 1. A straightforward way to generate multiple copies is the use of FWM in a nonlinear medium as shown in Fig. 1(a) [23]. The input signal is used as a pump in a degenerate FWM process. Two photons from the signal pump mix with the probe photons to generate the idlers (signal copies) at $f_{\text {idler }}=$ $2 f_{\text {sig }}-f_{\text {probe }}$ as depicted in Fig. 1(a). There is a need of $N$ probe lasers for $N$-fold multicasting. Using a low-dispersion, highly nonlinear medium, parametric gain can be obtained [24] with higher pump powers. In such a scheme, the probes are also modulated by the pump signal through the parametric gain. In this manner, only $N / 2$ pumps are required to generate $N$ outputmulticasted copies as depicted in Fig. 1(b). Another method is the use of multiple continuous wave (CW) pumps to generate idlers from an input signal using nondegenerate FWM [25]. This method uses $N / 2$ pumps to generate $N-1$ multicasted copies as depicted in Fig. 1(c). Since it uses a nondegenerate FWM setup, it can support phase-modulated signal multicasting as opposed to the schemes described in Fig. 1(a) and (b). Since methods in (a) and (b) are based on degenerate mixing, no spectral inversion (phase conjugation) takes place in the wavelength conversion. However, in Fig. 1(c) both phase conjugated and non-conjugated output copies are generated [25], and this needs to be tracked carefully for any following processes for complex operations, such as optical delays and buffers [26]. Another method is to use supercontinuum generation in a nonlinear medium as in Fig. 1(d). There is typically pulsewidth requirements to generate a wide output spectrum that can be combined with a periodic filter to slice the supercontinuum into multiple output channels [27], [28]. This method has also been shown to support the phase-modulated formats for low input powers [29]. 


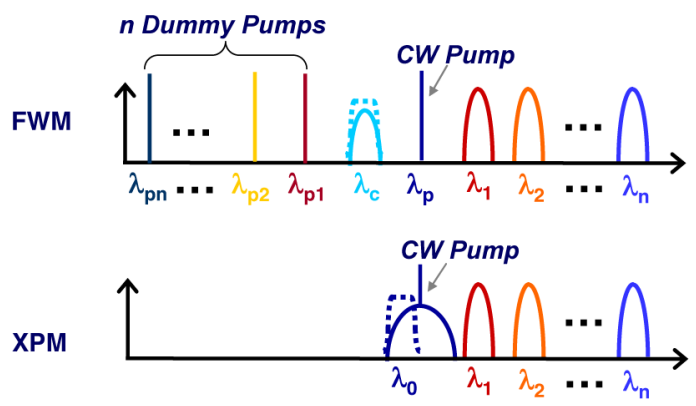

Fig. 2. Concept of WDM-to-TDM multiplexing. (a) Time domain. (b) Spectra comparison of XPM-based and FWM-based multiplexing.

In addition to these methods, several other techniques have been explored. These include XPM-based methods, where phase-modulated side lobes of a CW pump are filtered [30] using half as many probe pumps, and cross-gain modulation and cross-absorption modulation-based methods, where the gain (absorption) modulation in a semiconductor transfers the data to $\mathrm{CW}$ lasers using $N$ pumps [31].

\section{B. WDM-to-TDM Multiplexing}

Optical fiber communication systems are characterized by their extremely high transmission capacity. With high bandwidth and on-demand applications continuing to emerge, nextgeneration core optical networks will require significant improvements in capacity and reconfigurability [32]. Therefore, optimization of network usage may require efficient sharing of this high bandwidth among lower rate users. One popular networking approach is to time multiplex many channels together. Moreover, given that the lower speed channels will likely exist on different wavelengths in a WDM system, it is beneficial to envision wavelength, converting different low-rate channels onto a single-wavelength high-rate channel.

Typical approaches in doing WDM-to-TDM multiplexing include the use of FWM [33], XPM in HNLFs [34], or cSHG/DFG in PPLNs [35]. Fig. 2 gives a comparison of the FWM approach and the XPM approach. If we consider $N$-fold WDM-to-TDM multiplexing, $N+1$ pumps will be needed for the FWM case. In the XPM case, only one CW pump is required. The CW pump is phase modulated by the intensity of the $N$ WDM signals, and the required optical bandwidth is approximately half of the FWM case, as shown in Fig. 2. Subsequently, offset filtering, which serves as a phase-to-intensity converter, can be used to obtain the multiplexed signal. Due to the Kerr-effect-based nature of this multiplexing method, the applications are limited to intensity-modulated signal. For the case of FWM, phase coherence between within each tributary will be preserved due to nondegenerate FWM. However, phase coherence would be difficult to establish between the tributaries. On the other hand, since the XPM-based method is seeded from a CW source, the multiplexed signal will be phase coherent. We can see that XPM-based processes has the advantages of high efficiency in terms of optical bandwidth and a reduced number of pumps for WDM-to-TDM conversion.

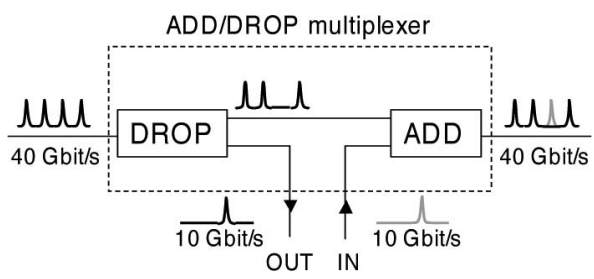

Fig. 3. Conceptual block diagram of an add/drop multiplexer.

\section{Add-Drop Multiplexing}

Single-channel extraction, clearing, and insertion from timeinterleaved optical signals is a key feature for networking operation in WDM/OTDM hybrid transmission systems.

Semiconductor devices are the most common candidates to perform this operation, thanks to their compactness, ease of integration, wide optical bandwidth, and high nonlinear coefficient [36], [37]. Nevertheless their characteristic response times limit the maximum bit rate of the signal to be processed.

On the other hand, optical fiber exhibits very fast dynamics of the Kerr effects. Add-drop multiplexers exploiting fibers can be based on a Kerr shutter [38] and separately carry out the extraction and insertion functionalities of a channel from a timeinterleaved optical frame, as shown in Fig. 3. Channel extraction can be obtained via polarization rotation through XPM using pump pulses at the tributary bit rate that coincide in time with the channel to be dropped. Ultrafast add/drop multiplexers have been demonstrated using nonlinear optical fibers up to $640 \mathrm{~Gb} / \mathrm{s}$ [39], [40]. Specialty nonlinear fibers allow for a reduction in the fiber length, down to $1 \mathrm{~m}$, with advantages in terms of stability and compactness [41].

The solution presented in [40] consists of using a nonlinear polarization-rotating loop, which is a looped version of the Kerr shutter. Similarly, the Add and Drop operations are performed separately. The $640-\mathrm{Gb} / \mathrm{s}$ speed operation has been reported also using PPLN waveguides [42].

Add and Drop operations can be carried out in a PPLN waveguide operating in a two-pump configuration using the parametric depletion effect. The depletion effect can be utilized on the signal or on clock signal such that either a polarity-inverted or noninverted demultiplexed signal can be achieved. The depletion effect is shown to be phase coherent and compatible with advanced modulation formats [43]. Note that cSFG/DFG introduce a broadening effect on the converted idler pulsed signal, due to chromatic dispersion, during the interaction between signals in different spectral regions. This distortion limits the speed of operation to $\sim 320 \mathrm{~Gb} / \mathrm{s}$ [44], [45]. On the other hand, parametric depletion introduces little distortion on the pumps enabling 640-Gb/s add/drop operations and beyond [45], [46]. In all the mentioned add/drop implementations, a single pump signal is required.

\section{Wavelength Exchange}

Robust data manipulation in the space, time, polarization, and wavelength domains might be valuable for superior network performance [46]. A desirable goal of optical signal processing 


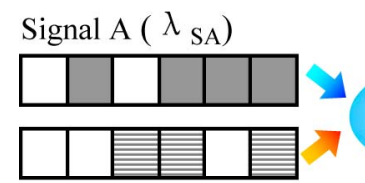

Signal B $\left(\lambda_{\mathrm{SB}}\right)$

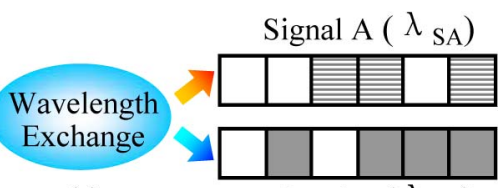

(a)
Signal B ( $\left.\lambda_{\mathrm{SB}}\right)$
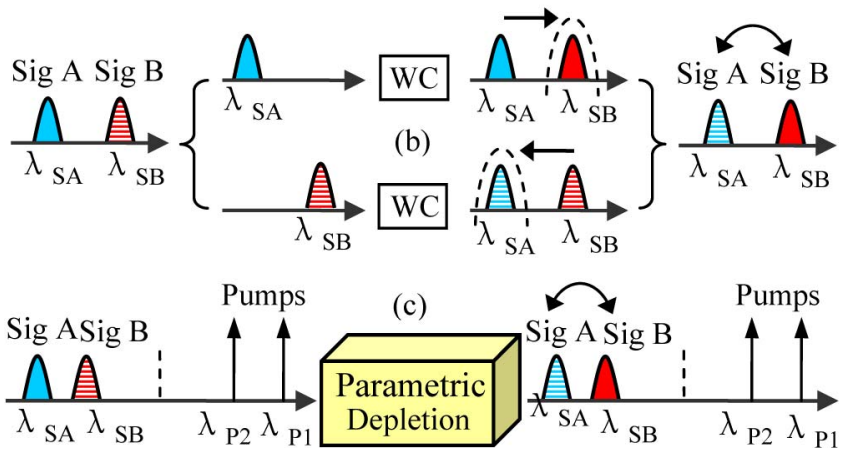

Fig. 4. (a) Concept of wavelength exchange. (b) Wavelength exchange by separate WCs. (c) Wavelength exchange by parametric depletion in single nonlinear device.

would be to efficiently utilize nonlinearities in the wavelength domain such that the data between two different wavelengths can be "exchanged," i.e., swapped, using single nonlinear processes in a single device. Wavelength exchange is a wavelength-domain data manipulation enabling the swapping of data between two different wavelengths, as illustrated in Fig. 4(a). One straightforward way, as shown in Fig. 4(b), is to use two separate wavelength converters (WCs) with one performing the wavelength conversion from signal A to signal B, and the other from signal $B$ to signal A. Other methods of wavelength exchange included the use of an optical parametric loop mirror [47] and 2-D nonlinear photonic crystal [48]. Toward single-device operation, another simple way of wavelength exchange is to explore the parametric depletion effects in a nonlinear device including a piece of HNLF [49]-[53] or a PPLN waveguide [54]-[56]. Nondegenerate FWM $\left(\chi^{(3)}\right)$ in a HNLF and cascaded second-order nonlinearities $\left(\chi^{(2)}: \chi^{(2)}\right)$ in a PPLN waveguide are potential choices. As shown in Fig. 4(c), due to the parametric depletion effects [57], [58] the data carried by signal A is depleted and converted to the wavelength of signal B and the vice versa. This enables single-device-based wavelength exchange. Parametric depletion effect can support phase-modulated formats and the converted signals are not spectrally inverted.

\section{RECENT ADVANCES FOR ENERGY-EFFICIENT OPTICAL SIGNAL PROCESSING}

We have generally discussed how optical signal processing functions can be achieved using nonlinear effects in various material platforms. In this section, we discuss energy efficiency in more details based on specific experimental demonstrations.

\section{A. Tunable Fold-Multicasting of ON-OFF Keying Signals Using Supercontinuum Generation}

Optical signal processing can be quite valuable for reducing optical-electrical conversions in straightforward functional op-

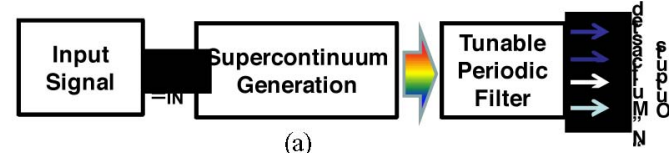

(a)

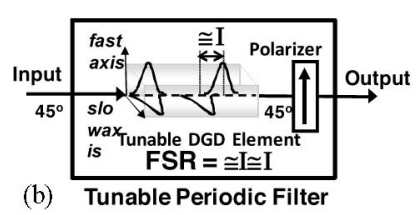

(c)

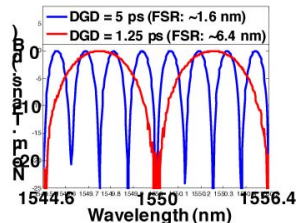

Fig. 5. (a) Conceptual block diagram of multicasting via supercontinuum. (b) Realization of the TPF in the polarization domain. (c) Transmission profiles of the TPF for different DGD values.

erations. For example, multicasting of data channels has potential utility for efficient system implementation of one-to-many processing functions, such as routing, parallel computing, and simultaneous critical data monitoring. We have investigated a tunable $N$-fold multicasting scheme that allows tunable temporal pulsewidth of $40-\mathrm{Gb} / \mathrm{s}$ channels using variable periodic slicing of a supercontinuum [59]. A supercontinuum is generated and then filtered with a periodic filter, comprising a tunable differential group delay (DGD) element and a polarizer, to generate multicasted copies of the original data. two-, four-, and eightfold multicasting of the 40-Gb/s return-to-zero ON-OFF keying (RZ-OOK) waveform with average penalties of $0.1,0.26$, and $0.44 \mathrm{~dB}$, respectively, at a $10^{-9}$ bit error rate (BER) are shown.

Typically, wave mixing or Kerr-effect-based multicasting approaches require at least half as many additional pump lasers for multicasted copies. The total optical energies needed scale with the input signal and pump powers [60] determined by the scheme used. For example, seven seed lasers were used for sevenfold multicasting of 10-Gb/s OOK signals using an electroabsorption (EA) modulator [31]. Also, 40-fold multicasting is achieved in an HNLF using 20 seed lasers [24] and optical parametric amplification at $40 \mathrm{~Gb} / \mathrm{s}$. Furthermore, FWM with three pumps was used for sixfold multicasting in Bi-HNLF [25] for 20-Gb/s ASK- differential phase-shift keying (DPSK) signals. Another method used XPM in an HNLF for $40-\mathrm{Gb} / \mathrm{s}$ OOK signals. It is also demonstrated in Si waveguides using FWM [61].

The conceptual block diagram of the supercontinuum technique is shown in Fig. 5(a). A supercontinuum is generated from an input signal and then "sliced" by a tunable periodic filter (TPF) to achieve multicasting. A commercially available tunable DGD element and a polarizer are used to realize the TPF. As shown in the Fig. 5(b), in the TPF, the signal can be decomposed into principal polarization states. A relative delay is induced between the states by the tunable DGD element. The two polarizations are then recombined in a polarizer resulting in a delay-line interferometer in polarization domain with the transfer function $(1+\cos (2 \pi f \Delta \tau+\theta))$, where $\Delta \tau$ is the delay and $\theta$ is the relative phase difference between the two states of polarization [62]. Fig. 5(c) shows the transmission spectra for two settings of the DGD $(\Delta \tau)$ element, which is tuned to change the number of output channels (multicasting order), since the free spectral range (FSR) is equal to $1 / \Delta \tau$. Furthermore, the 


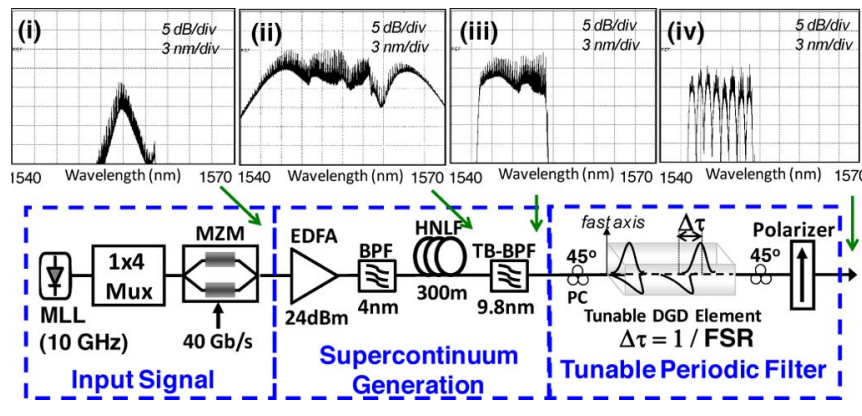

Fig. 6. Experimental block diagram for supercontinuum-based multicasting along with experimental spectra for different stages. BPF: bandpass filter; MZM: Mach-Zehnder modulator; PC: polarization controller; MLL: modelocked laser.
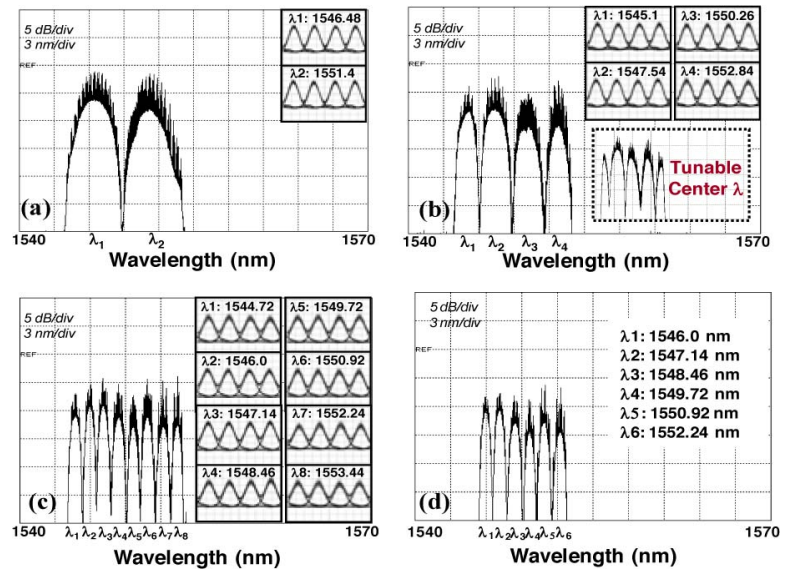

Fig. 7. Experimental spectra for different orders of multicasting: (a) Twofold, (b) fourfold, (c) eightfold, and (d) sixfold by changing the TB-BPF bandwidth.

center wavelength for the passbands can be changed by adjusting the polarization controllers in the TPF to change the relative phase $\theta$ between the polarization states.

The experimental block diagram of our technique is shown in Fig. 6 along with the experimental spectra at various stages. A short-pulse laser (at $1554.9 \mathrm{~nm}$ ) with a repetition rate of $10 \mathrm{GHz}$ and pulsewidths of $\sim 2 \mathrm{ps}$ is used at the transmitter. The modulated signal is then amplified and sent through a $300-\mathrm{m}$ HNLF with a zero-dispersion wavelength (ZDW) at $\sim 1561 \mathrm{~nm}$ for supercontinuum generation as shown in Fig. 6(graph ii). A portion of the generated supercontinuum is filtered with a tunable-bandwidth bandpass filter (TB-BPF) set to $\sim 9.8 \mathrm{~nm}$ bandwidth [see Fig. 6(graph iii)]. Later, the filter bandwidth is tuned to change the multicasting order. The selected supercontinuum section is then channelized with the TPF as shown in Fig. 6(graph iv). The tunable DGD element used in the experiment is a commercially available tunable DGD emulator (JDSU PE4).

Experimental spectra of four different multicasting orders, twofold (a), fourfold (b), eightfold (c), and (d) sixfold, are shown in Fig. 7, along with the eye diagrams of the multicasted channels. The DGD values are set to $1.65,3.3$, and $6.6 \mathrm{ps}$ for Fig. 7(a)-(c), respectively. This corresponds to $\sim 4.8-, \sim 2.4-$, and $\sim 1.2$-nm-wide output channels. With the 6.6-ps setting,
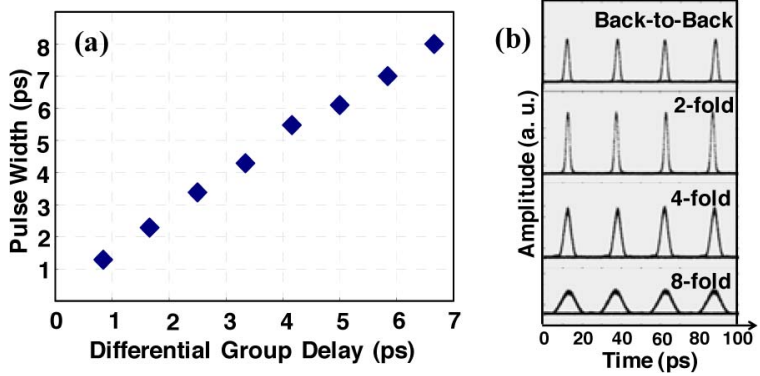

Fig. 8. (a) Output pulsewidth versus DGD (multicasting order). (b) Eye diagrams for different multicasting orders captured by an optical sampling scope.
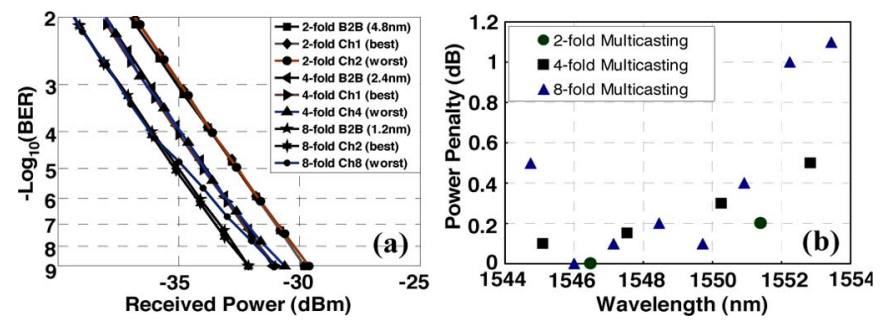

Fig. 9. (a) BER results and (b) Received power penalties (at $10^{-9} \mathrm{BER}$ ) for different multicasting orders.

the TB-BPF bandwidth is changed to $\sim 7.4 \mathrm{~nm}$ resulting in six 1.2-nm output channels, as shown in Fig. 7(d). The inset in Fig. 7(b) shows the tunability of the center wavelengths of the multicasted channels by tuning the $\theta$ for the fourfold multicasting case.

Pulsewidth versus DGD for a fixed supercontinuum portion $(9.8 \mathrm{~nm})$ along with the eye diagrams [see Fig. 8(b)] from an optical sampling scope is shown in Fig. 8(a). The DGD (1/FSR) provides an almost linearly change in output pulsewidth. The measured pulsewidths are $\sim 2.2,4.3$, and 8 ps for the two-, four-, and eightfold multicasting cases, respectively.

BER measurements are obtained for all multicasted channels for two-, four-, and eightfold multicasting by filtering each channel with a 4.8-, 2.4-, and 1.2-nm filter, respectively. Fig. 9(a) shows the BER curves for the best and worst case performances along with the back-to-back (B2B) performance. Three different B2B curves are obtained by filtering the transmitted signal with the same filter used to extract the multicasted channels. Fig. 9(b) shows the power penalty (at a BER of $10^{-9}$ ) with respect to the relevant $\mathrm{B} 2 \mathrm{~B}$ performance. An average penalty of $0.1,0.26$, and $0.44 \mathrm{~dB}(0.2,0.5$, and $1.1 \mathrm{~dB}$ maximum $)$ exists for the two-, four-, and eightfold multicasting cases, respectively.

The optical energy consumption of the supercontinuum-based multicasting method is $\sim 0.8 \mathrm{pJ} / \mathrm{bit}$ per multicasted output channel. It can also be extended to phase-modulated schemes as in ninefold multicasting of DPSK signals reported in [29] with an optical energy consumption of $\sim 0.2 \mathrm{pJ} / \mathrm{bit} / \mathrm{channel}$, where the complete supercontinuum is utilized. 


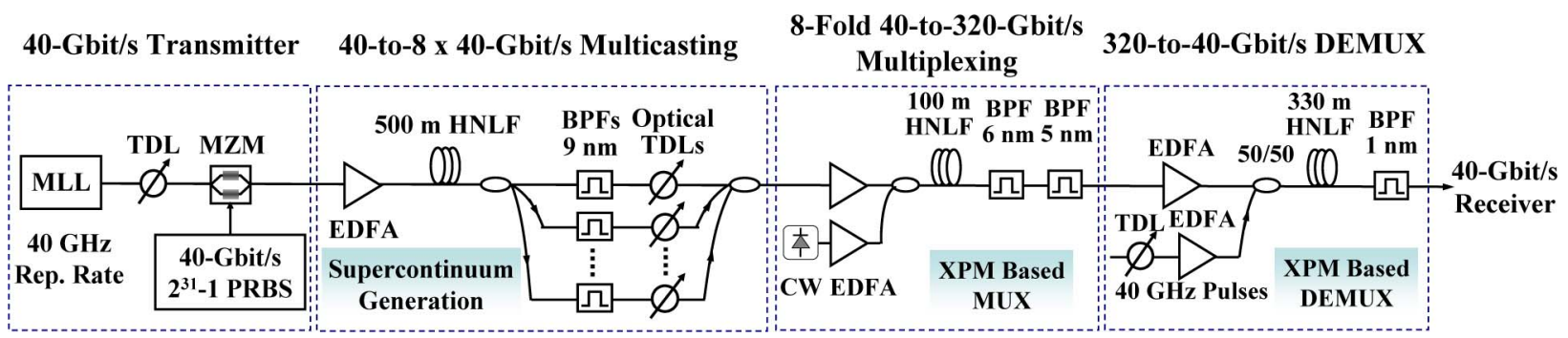

Fig. 10. Experimental setup for 40-160-Gb/s WDM-to-TDM conversion. TDL: tunable delay line; PC: polarization controller; MZM: Mach-Zehnder modulator; BPF: bandpass filter; CW: continuous wave; MUX: multiplexing; DEMUX: demultiplexing.

\section{B. Eightfold 40-320-Gb/s Multiplexing Using Cross-Phase Modulation in HNLF}

Multiuser networks tend to manipulate (i.e., multiplexing, demultiplexing) multiple lower data-rate channels in order to facilitate efficient routing and to optimally utilize links of differing capacities. This granularity adds to the usefulness of a network, and optics enables such granularity in the wavelength domain. In this section, we show the WDM-to-TDM multiplexing of eight $40-\mathrm{Gb} / \mathrm{s}$ WDM channels to one single $320-\mathrm{Gb} / \mathrm{s}$ channel using XPM.

Earlier results for high-speed time multiplexing include multiplexing lower speed, same wavelength channels into a single $\sim 1$ - Tb/s signal such that optical delays are arranged to interleave the bit streams without any wavelength conversion involved [63]. WDM to OTDM approaches have used semiconductor optical amplifiers (SOAs) [64] and EA modulators [65], with bit rates up to $60 \mathrm{~Gb} / \mathrm{s}$ achieved. Results were also shown using HNLFs based on FWM [33], supercontinuum generation [66], [67] and XPM [34], [67]. In general, there are always significant technical challenges to achieve high bit rates with high performance. XPM-based processes for WDM-to-TDM conversion has the advantages of high efficiency in terms of bandwidth and a reduced number of pumps. In addition, XPMbased conversion depends just on the signal envelope, which results in the multiplexed signal maintaining the phase coherence of the pump [68].

Fig. 10 shows the experimental setup for phase-coherent eightfold 40-320-Gb/s multiplexing. Fig. 11(a) shows the generated supercontinuum after the 500-m HNLF. To obtain multicasted copies after supercontinuum generation, eight filters with $\sim 9 \mathrm{~nm}$ bandwidth are used, with center wavelengths from $\sim 1551$ to $1565 \mathrm{~nm}$ in steps of $2 \mathrm{~nm}$. A pulsewidth of $\sim 1.2 \mathrm{ps}$ is obtained for each channel. Note that the purpose of the multicasting stage is to emulate the eight channels. They overlap in the frequency domain, but do not overlap in the time domain. For the practical implementation of WDM-to-TDM multiplexing, in order to obtain the RZ signals with short pulsewidth, optical sampling would possibly be required to convert the original data channels. Shown in Fig. 11(b) is the optical spectrum after the XPM-based multiplexing stage. The combined four multicasted copies can be seen on the right- hand side. Note that they overlap in the frequency domain, but do not overlap in the time domain. The 320-GHz tones in the broadened pump spectrum show the successful multiplexing to $320 \mathrm{~Gb} / \mathrm{s}$. The $\mathrm{CW}$

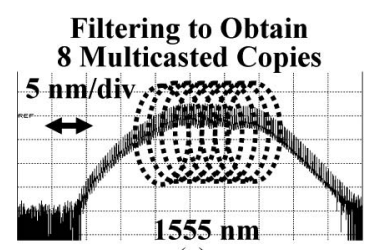

(a)

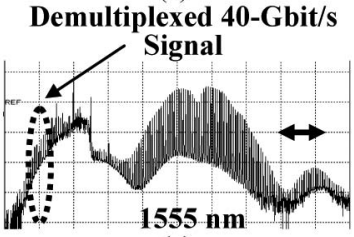

(c)

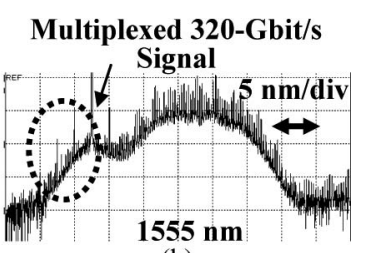

(b)

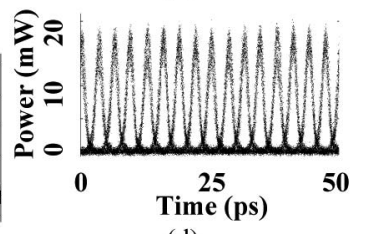

(d)
Fig. 11. (a) Optical spectra of the multicasting stage, (b) optical spectra of the XPM-based multiplexing stage, (c) optical spectra of the XPM based demultiplexing stage, and (d) eye diagrams of the $320-\mathrm{Gb} / \mathrm{s}$ multiplexed signal.
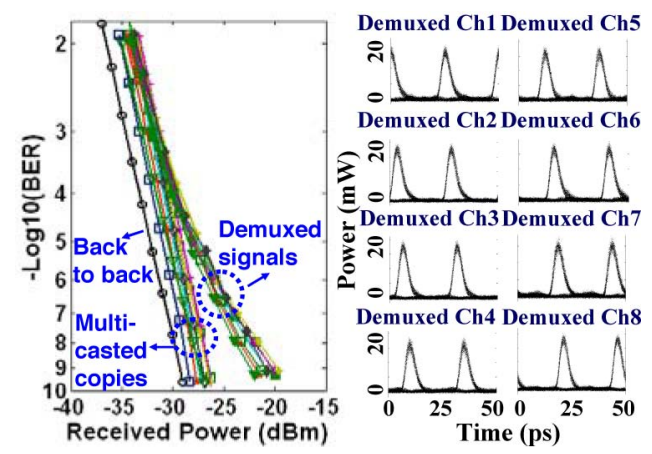

Fig. 12. BER performance of the $40-\mathrm{Gb} / \mathrm{s}$ tributaries of the multiplexed $320-\mathrm{Gb} / \mathrm{s}$ signal.

pump power into the $100-\mathrm{m}$ HNLF is $\sim 23 \mathrm{~dB} \cdot \mathrm{m}$ and the total average power of the eight signals is $\sim 18 \mathrm{~dB} \cdot \mathrm{m}$, which gives the optical energy consumption of the multiplexing approach to be $\sim 0.8 \mathrm{pJ} / \mathrm{bit}$. Cascaded filters of bandwidths 6 and $5 \mathrm{~nm}$ are used to filter out the multiplexed 320-Gb/s signal. Subsequently, the $320-\mathrm{Gb} / \mathrm{s}$ signal is demultiplexed to $40 \mathrm{~Gb} / \mathrm{s}$ and Fig. 11(c) shows the spectrum after demultiplexing. Eye diagram of the multiplexed $320-\mathrm{Gb} / \mathrm{s}$ signal with a pulsewidth of $\sim 1.8 \mathrm{ps}$ is shown in Fig. 11(d).

Fig. 12 shows the BER performance of the eight multicasted copies and eight demultiplexed tributaries. The eye diagrams for each demultiplexed $40-\mathrm{Gb} / \mathrm{s}$ tributary are also given. An average penalty of approximately $2 \mathrm{~dB}$ at $10^{-9} \mathrm{BER}$ is observed for the 


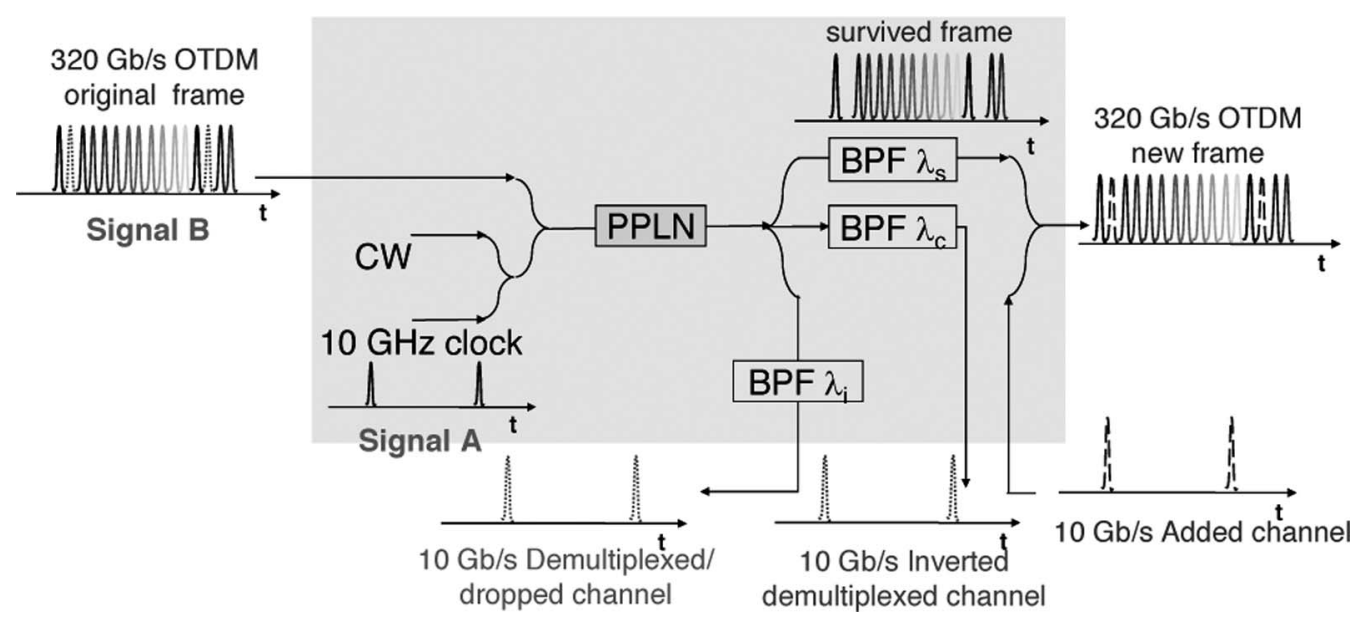

Fig. 13. Concept of Add/Drop operations based on a PPLN waveguide.

multicasting copies when compared to the $40-\mathrm{Gb} / \mathrm{s}$ B2B performance. An extra penalty of approximately $7 \mathrm{~dB}$ is observed after the eightfold $40-320-\mathrm{Gb} / \mathrm{s}$ multiplexing process and the $320-40-\mathrm{Gb} / \mathrm{s}$ demultiplexing, mainly due to the pulse broadening through the fiber, optical filters and erbium-doped fiber amplifiers, and the slow phase drift of the signal and sampling pulse train induced by fluctuations of the HNLF length [69].

The optical energy consumption for XPM-based 40-320$\mathrm{Gb} / \mathrm{s}$ multiplexing is $\sim 0.8 \mathrm{pJ} / \mathrm{bit}$, which can be further decreased in media with higher nonlinearities for more effective XPM [70]. The silica-HNLFs used in the demonstration can be replaced by special HNLFs, having much shorter lengths and thus reducing the phase drift significantly [71].

\section{Add/Drop Multiplexing Based on Parametric Depletion in PPLN Waveguide}

Channel extraction, clearing from time-interleaved optical signals, and new single-channel insertion in the time domain are the key features for efficient operation in WDM networks. Earlier, SOAs [36] and electroabsorption modulators [37] have been used to perform add/drop operations up to $160 \mathrm{~Gb} / \mathrm{s}$, whereas nonlinear optical fibers enabled add/drop operations up to $640 \mathrm{~Gb} / \mathrm{s}$ [39], [40]. Recently, PPLN waveguides have drawn lots of attention for all-optical signal processing due to their ultrafast dynamics, high efficiency, and compactness. PPLN waveguides have been used to obtain $160-\mathrm{Gb} / \mathrm{s}$ OTDM to WDM conversion [72] and wavelength conversion [73] exploiting SFG/DFG. Demonstrations of $160-\mathrm{Gb} / \mathrm{s}$ half-adder, halfsubtractor, OR/XOR [74] operations by combining the parametric depletion effect with SFG/DFG were also reported. Moreover, recent results show the possibility of using PPLN waveguides at room temperature to avoid the energy consumption due to temperature control [75]. PPLN waveguides can provide a large number of nonlinear functions when operating in a two-pump configuration as shown in Fig. 13. Two pumps (A and B) can nonlinearly interact through SFG defined by the quasi-phase matching (QPM) condition. The generated signal simultaneously interacts with a $\mathrm{CW}$ light to produce an idle signal in the C-band through the DFG process. Looking at A, B, and the

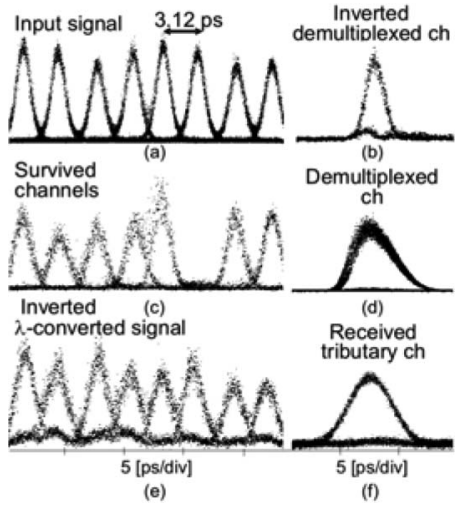

Fig. 14. Eye diagrams of the involved signals in Add/Drop operations.

idle signal at the output of the PPLN waveguide, we can obtain different nonlinear operations.

The PPLN in the experiment is fabricated by the reverseproton-exchange technique. Input peak powers of 27 and $18 \mathrm{~dB} \cdot \mathrm{m}$ for the clock and OTDM signal, respectively, allow for an optimized parametric depletion of the OTDM signal. The parametric depletion optimization on the clock signal requires exchanged peak power values. The $\mathrm{CW}$ input power is $\sim 25 \mathrm{~dB} \cdot \mathrm{m}$ for both cases. Finally a $320-10-\mathrm{Gb} / \mathrm{s}$ optical demultiplexer based on XPM effect in an HNLF has been used to test the performance of the $320-\mathrm{Gb} / \mathrm{s}$ add/drop multiplexing. Fig. 14 shows the eye diagrams of the involved signals. The $320-\mathrm{Gb} / \mathrm{s}$ OTDM input signal has a pulsewidth of $1.7 \mathrm{ps}$, as shown in Fig. 14(a), while the 10- and 320-GHz input clocks are 2 and 2.4 ps, respectively. For the demultiplexing operation, we use a $10-\mathrm{GHz}$ clock synchronized with the tributary channel to be demultiplexed. This way, an inverted and a noninverted replica of the demultiplexed channel are observed, respectively, as shown in Fig. 14(b) and (d). If we optimize the parametric depletion effect for the OTDM signal, the survived channels at the OTDM signal wavelength are extracted at the PPLN waveguide output. From Fig. 14(c), we can see that no severe distortions are observed in the eye diagram with respect to that of the input signal. 


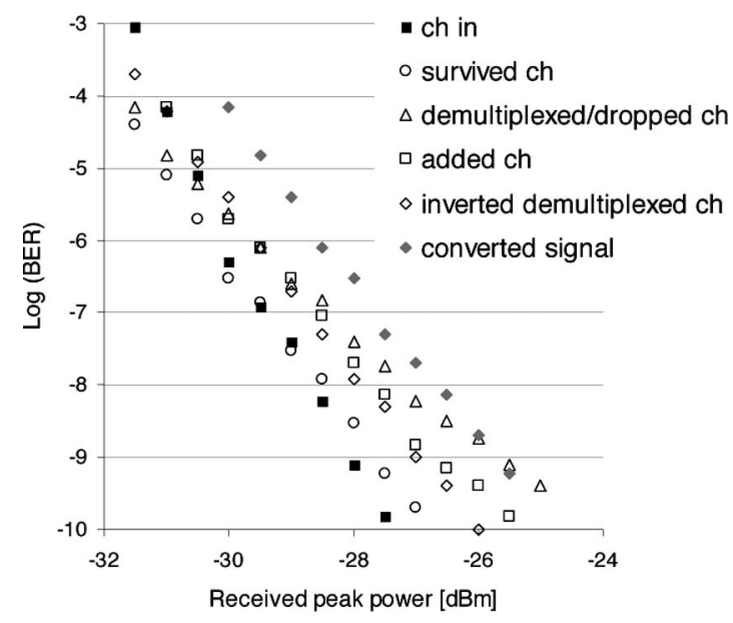

Fig. 15. 320-Gb/s BER measurement for Add/Drop operations.

In addition, 320-Gb/s inverted wavelength conversion, as mentioned earlier, can be obtained exploiting parametric depletion on a 320-Gb/s clock, as shown in Fig. 14(e). In this case, no distortions are evident and the converted signal presents almost the same pulse shape as the input clock. Finally, BER measurements at $320 \mathrm{~Gb} / \mathrm{s}$ have been carried out for all optical nonlinear subsystems, obtaining error-free operations in all cases. Fig. 14(f) shows the 10-Gb/s eye diagram of the received channel of the original $320-\mathrm{Gb} / \mathrm{s}$ OTDM frame after demultiplexing based on XPM in HNLF. Such demultiplexing introduces a penalty of less than $1 \mathrm{~dB}$, and for all nonlinear operations, the penalty is lower than $3 \mathrm{~dB}$, as shown in Fig. 15 .

The optical energy consumption for each add and drop operation involving parametric depletion effect is lower than $1 \mathrm{pJ} / \mathrm{bit}$. Drop operation based on SFG/DFG increases the optical energy consumption up to $2 \mathrm{pJ} / \mathrm{bit}$. The energy consumption is partially due to the high coupling loss of the PPLN used in the experiment $(\sim 4 \mathrm{~dB})$. New generation devices [76] with low coupling loss could greatly reduce the power value. Moreover, recent results show the possibility to use PPLN waveguides at room temperature avoiding the energy consumption of temperature control [75].

We successfully carried out $320-\mathrm{Gb} / \mathrm{s}$ time-domain demultiplexing, add/drop multiplexing, and wavelength conversion operations. The obtained energy efficiencies are still limited by the specific device used in the experiment, where it is about $2 \mathrm{pJ} / \mathrm{bit}$.

\section{PPLN-Assisted Time- and Channel-Selective Data Exchange Between WDM Channels}

Single-device single-stage-enabled wavelength exchange is a desirable feature for the efficient operation in WDM networks. We investigate time- and channel-selective optical data exchange between multiple WDM channels by exploiting the cascaded second-order nonlinear interactions in a PPLN waveguide. Two gated pumps are employed supporting both timeand channel-selective operations through the proper adjustment of the gated pump pulse duration and pump wavelengths. Using

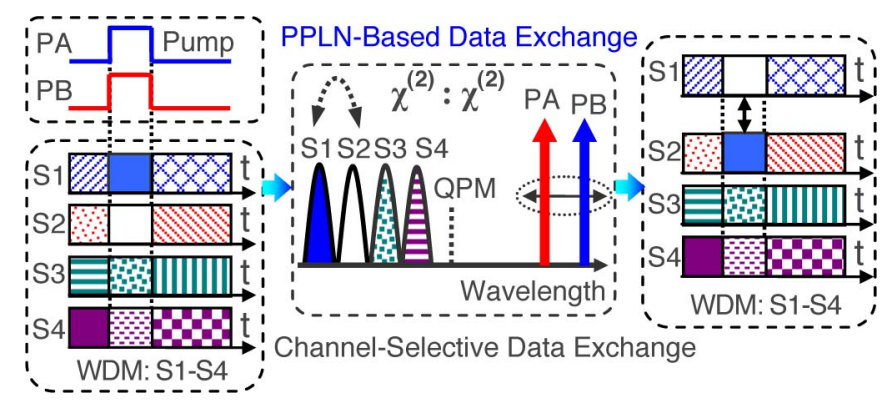

Fig. 16. Concept of PPLN-based time- and channel-selective optical data exchange between WDM channels.

a single PPLN waveguide, this method provides a simple way to implement data exchange between two channels of interest without touching other channels and introducing any additional spectrum. We demonstrate optical data exchange between two WDM channels with a power penalty of less than $1.5 \mathrm{~dB}$ at $10 \mathrm{~Gb} / \mathrm{s}$ and $3 \mathrm{~dB}$ at $40 \mathrm{~Gb} / \mathrm{s}$ at a BER of $10^{-9}$. Also, $40-\mathrm{Gb} / \mathrm{s}$ channel-selective optical data exchange between four WDM channels with a power penalty of $\sim 4 \mathrm{~dB}$ at a BER of $10^{-9}$ is achieved [54], [55].

Earlier, nondegenerate FWM in an HNLF was widely used for wavelength exchange [50]-[53]. In [49], [50], wavelength exchange between a $2.5-\mathrm{Gb} / \mathrm{s}$ modulation and a $10-\mathrm{Gb} / \mathrm{s}$ modulation was proposed and demonstrated using a 1-km-long dispersion-shifted HNLF. Further improvement was achieved in [51] showing tunable $(>15 \mathrm{~nm}) 10-\mathrm{Gb} / \mathrm{s}$ wavelength exchange with two pumps in the anomalous-dispersion region, which eliminated the performance degradation caused by Raman gain. In [52], HNLF-based byte-level wavelength exchange was investigated using square-wave-modulated pumps.

The conceptual diagram of our proposed PPLN-assisted time and channel-selective optical data exchange between WDM channels is shown in Fig. 16. Multiple WDM channels (S1-S4) and two synchronized gated pumps (PA and $\mathrm{PB}$ ) are coupled into a PPLN waveguide in which cSFG/DFG processes take place. The wavelength selectivity of the QPM condition allows selection of channels for data exchange by proper choice of the two pump wavelengths. For proper QPM of both cSFG/DFG processes, the two pump wavelengths are nearly symmetric to the two exchanged data wavelengths with respect to the QPM wavelength. For instance, as illustrated in Fig. 16, within the gated pump pulse duration, $\mathrm{PB}$ mixes with $\mathrm{S} 1$ to produce a sum frequency (SF) wave through the SFG process. Meanwhile, the SF wave interacts with PA to generate a new idler at the wavelength of $\mathrm{S} 2$ by the subsequent DFG process. During such parametric nonlinear interactions, S1 can be depleted [57], and converted to S2 by means of proper control of the pump powers. Similarly, PA and S2 participate in the SFG process to create a SF wave, which simultaneously interacts with PB to yield an idler at the wavelength of $\mathrm{S} 1$ via the DFG process. Consequently, it is expected to implement optical data exchange between $\mathrm{S} 1$ and S2 without the use of additional spectrum and touching other channels. 


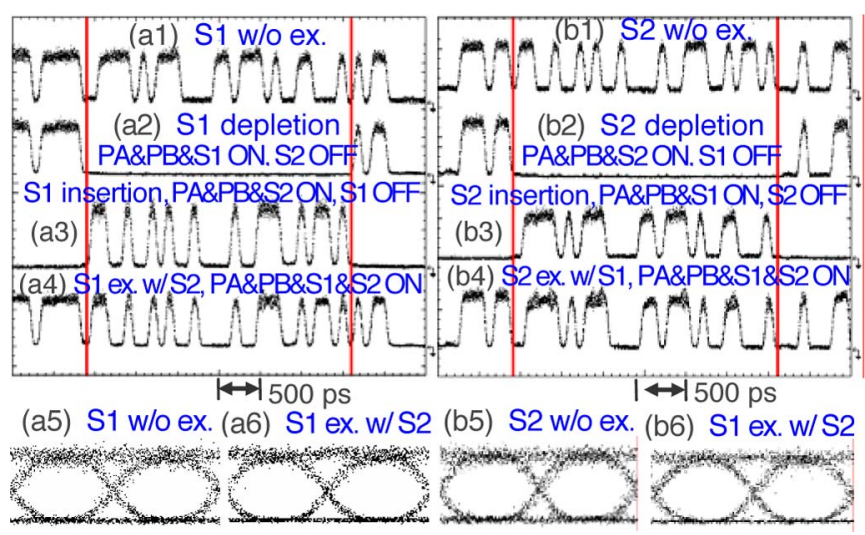

Fig. 17. Measured temporal waveforms [(a1-a4) and (b1-b4)] and eye diagrams [(a5), (a6), (b5), and (b6)] of 10-Gb/s data exchange.

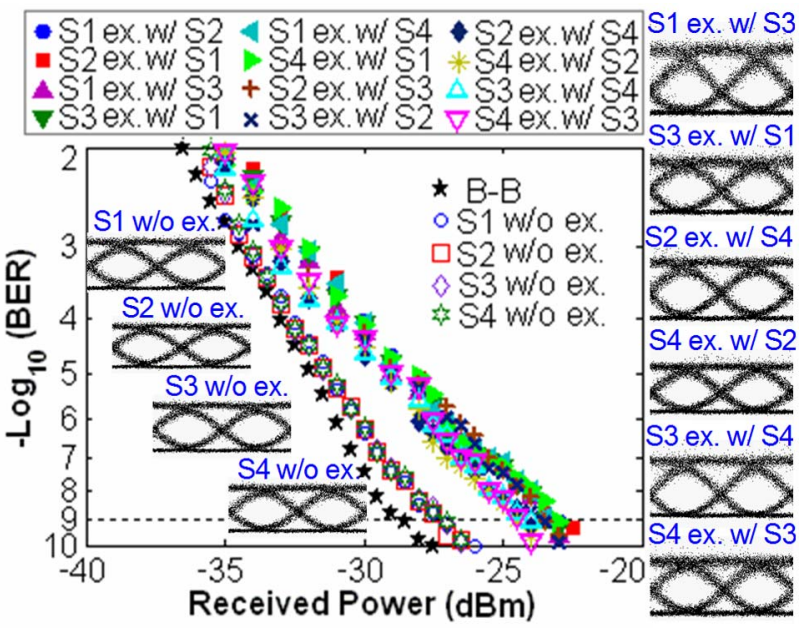

Fig. 18. Measured eye diagrams and BER performance of 40-Gb/s channelselective optical data exchange between four WDM channels.

We first demonstrate the optical data exchange between two $10-\mathrm{Gb} / \mathrm{s}$ signals. Two gated pumps with a duty cycle of $1 / 127$ and a pulse duration of $\sim 3.2 \mathrm{~ns}$ are employed. The average power of each signal and peak power of each pump coupled into the PPLN waveguide are about $4 \mathrm{~mW}$ and $1 \mathrm{~W}$, respectively. Fig. 17 displays the observed temporal waveforms and eye diagrams of data exchange. The time slots between the two straight lines correspond to the gated pump pulse duration in which optical data exchange occurs. For the $10-\mathrm{Gb} / \mathrm{s}$ operation, we obtain a power penalty of less than $1.5 \mathrm{~dB}$ at a BER of $10^{-9}$ with an optical energy consumption of $\sim 2.37 \mathrm{pJ} / \mathrm{bit}$.

We also investigate the PPLN-based $40-\mathrm{Gb} / \mathrm{s}$ optical data exchange between two signals. Two gated pumps with a duty cycle of 3/127 and pulse duration of $\sim 1.2 \mathrm{~ns}$ are adopted. The power penalty of $40-\mathrm{Gb} / \mathrm{s}$ data exchange is measured to be $\sim 3 \mathrm{~dB}$ with an optical energy consumption of $\sim 1.38 \mathrm{pJ} / \mathrm{bit}$.

We further demonstrate the PPLN-based channel-selective data exchange for multiple WDM channels at $40 \mathrm{~Gb} / \mathrm{s}$. Four WDM channels (S1: $1535.5 \mathrm{~nm}, \mathrm{~S} 2: 1539.4 \mathrm{~nm}, \mathrm{~S} 3: 1543.3 \mathrm{~nm}$, $\mathrm{S} 4: 1547.2 \mathrm{~nm}$ ) are employed in the experiment. It is possible to perform a channel-selective data exchange by simply tuning the wavelength of the two pumps. Fig. 18 displays the measured typ- ical eye diagrams and BER performance for channel-selective data exchange between WDM channels. The power penalty of $40-\mathrm{Gb} / \mathrm{s}$ channel-selective exchange is estimated to be less than $4 \mathrm{~dB}$.

In view of reported experiments [49]-[56], PPLN and HNLF might be advantageous in terms of using only a single device for wavelength exchange based on the parametric depletion effect. As a key function of data traffic grooming, wavelength exchange can enhance the flexibility of optical networks. In particular, toward the robust grooming exchange, PPLN-/HNLF- assisted wavelength exchange is also available for different modulation formats and different granularities (entire data [49]-[51], [53], byte-level groups of bits [52], [54], [55], and tributary channels [56]).

\section{INTEGRATED WGS FOR POTENTIALLY GREEN SIGNAL PROCESSING}

Integrated photonics has attracted a great deal of attention in recent years not only because it allows for more cost-effective production and easier packaging, but also because smaller chip size assists in realizing faster electro-optic interaction and less energy-consuming photonic devices to facilitate energyefficient information technology [77]. Integrated photonics can potentially enable sophisticated optical signal processing subsystems by cascading many basic functional components on a single chip. This in turn imposes stricter requirements on the power consumption of each functional device to avoid significantly increased power density on limited chip area.

In integrated photonics, nonlinearity again lays a foundation for signal processing, and three factors become critical in determining nonlinear efficiency: optical power, nonlinear coefficient $\gamma$, and nonlinear interaction length. Since the integrated nonlinear media is typically much shorter in length than optical fibers, the nonlinear coefficient has to be extremely high [78] to effectively reduce the energy consumption. Essentially, the nonlinear coefficient relies on the material's nonlinear index $n_{2}$ and effective mode area $A_{\text {eff }}$. Many research efforts have been made in recent years to develop new extremely high nonlinear materials and to design novel WG structures with enhanced light confinement.

Highly nonlinear integrated WGs can be composed of silicon [79], silicon nitride [80], Si nanocrystals (Si-nc or Si-rich oxide) [81], [82], III-V compound semiconductors [83], chalcogenide glasses [84], [85], to name a few. Nonlinear index $n_{2}$ ranges from $10^{-19}$ to $10^{-17} \mathrm{~m}^{2} / \mathrm{W}$, orders of magnitude higher than silica. On the other hand, there has been several demonstrations able to confine light in a tiny spot. An introduction of a slot structure [86] takes advantage of the electric field discontinuity at the material interfaces and makes low-index highly nonlinear materials very useful for confining light, opening an opportunity to further reduce the effective mode area $A_{\text {eff }}$ to $0.01 \mu \mathrm{m}^{2}$. Benefiting from the aforementioned advantages, the integrated WGs have been made highly nonlinear, as summarized in Table I.

The high index contrast in the integrated photonics platform not only enables strong light confinement, but also provides great tailorability of chromatic dispersion that plays a critical 
TABLE I

COMPARISON OF OPTICAL PROPERTIES OF VARIOUS OPTICAL MEDIA

\begin{tabular}{|c|c|c|c|c|}
\hline Material & Type & $\begin{array}{c}\gamma \\
\left(\mathrm{W}^{-1} \cdot \mathrm{m}^{-1}\right)\end{array}$ & $\begin{array}{c}\text { Dispersion } \\
(\mathrm{ps} /(\mathrm{nm} \cdot \mathrm{km}))\end{array}$ & Ref \\
\hline $\mathbf{S i O}_{\mathbf{2}}$ & Fiber & 0.017 & -0.1 & {$[84]$} \\
\hline $\mathbf{B i}_{2} \mathbf{O}_{3}$ & Fiber & 1.36 & -260 & {$[84]$} \\
\hline $\mathbf{A s}_{\mathbf{2}} \mathbf{S}_{\mathbf{3}}$ & Strip WG & 10 & Tailorable & {$[85]$} \\
\hline $\mathbf{S i}$ & Strip WG & 181 & Tailorable & {$[87]$} \\
\hline $\mathbf{S i}-\mathbf{n c}$ & Slot WG & 2874 & Tailorable & {$[87]$} \\
\hline
\end{tabular}
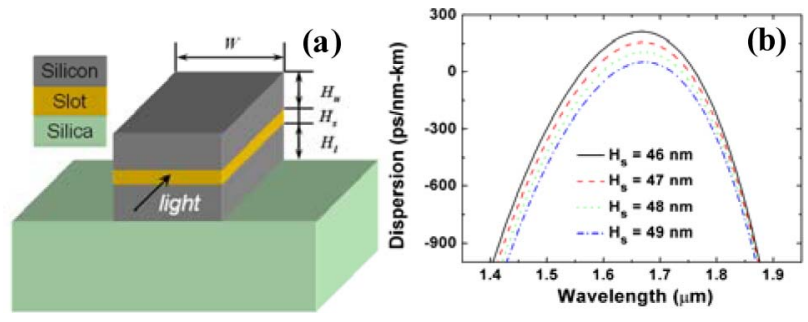

Fig. 19. (a) Integrated slot WG with silicon layers surrounding a highly nonlinear silicon nanocrystal slot layer. (b) For highly nonlinear Si nanocrystal slot WGs, dispersion profiles change with slot height $H_{s}$.

role in determining nonlinear efficiency as well. Overall dispersion is dominated by WG dispersion, making it possible to achieve low dispersion over a wide wavelength range and desirable ZDW [87]. We describe a highly nonlinear Si-nc slot WG, with chromatic dispersion designed for nonlinear applications. The WG structure is shown in Fig. 19(a). A horizontal slot is surrounded by two silicon layers with air cladding. A $2-\mu \mathrm{m}-$ thick buried oxide layer serves as WG substrate. A large fraction of vertically polarized quasi-TM mode can be confined in the slot layer [86] due to the discontinuity of its electric field at the interfaces of the slot and the silicon layers we choose WG width $W=500 \mathrm{~nm}$, and upper silicon height $H_{u}$ equal to lower silicon height $H_{l}$ is $180 \mathrm{~nm}$, while slot thickness $H_{s}$ is $47 \mathrm{~nm}$. Fig. 19(b) shows a dispersion profile within $\pm 160 \mathrm{ps} /(\mathrm{nm} \cdot \mathrm{km})$ obtained over a 244-nm wavelength range, from 1539 to $1783 \mathrm{~nm}$. There are two ZDWs at 1580 and $1751 \mathrm{~nm}$, respectively. Calculated nonlinear coefficient $\gamma$ is $2874 /(\mathrm{W} \cdot \mathrm{m})$. The obtained dispersion is not as flat as in silica fibers due to the strong WG dispersion, but the accumulated dispersion in the nonlinear processes is essentially quite low due to the short device length.

Although the integrated WGs exhibit a great potential for "green" optical signal processing, some problems, such as twophoton absorption could be quite challenging [86]. Furthermore, high power densities in such small footprint devices should be taken into consideration. Such exotic WGs with high-performance metrics are promising devices in order to achieve on-chip, easy-to-integrate, and "green" optical signal processing.

\section{CONCLUSION}

Optical signal processing techniques based on (silica and bismuth) HNLFs, semiconductor-based electro/optical devices, and WGs, such as PPLNs, Silicon, and chalcogenide have been discussed. These techniques differ in the nonlinearities used for the realization of the processing function. Along with the device specifications, careful choice of the nonlinearity exploited plays an important role in determining the number of necessary pumps and the pump powers contributing to the optical energy consumption. Limiting the number of required pumps reduces coupling loss, the number of components in the system, and can improve the overall optical energy per bit requirement. Furthermore, the high bandwidth of optics and the ability to process an entire channel without "touching" each bit allows the optical energy per bit to decrease with increasing data rates. Additionally, novel optical materials and the development of WGs with extreme nonlinearities may provide even lower optical energy consumptions at data rates far greater than $100 \mathrm{~Gb} / \mathrm{s}$.

\section{ACKNOWLEDGMENT}

The authors would like to thank Fejer and Langrock at Stanford University for providing the PPLN waveguides.

\section{REFERENCES}

[1] J. Marti and K. Williams, "Is all-optical processing green?" presented at Workshop OFC 2010, San Diego, CA.

[2] R. S. Tucker, "A green internet," in Proc. IEEE Lasers Electro-Opt. Soc. Annu. Meeting, 2008, pp. 4-5.

[3] H. G. Weber, S. Ferber, M. Kroh, C. Schmidt-Langhorst, R. Ludwig, V. Marembert, C. Boerner, F. Futami, S. Watanabe, and C. Schubert, "Single channel 1.28 Tbit/s and 2.56 Tbit/s DQPSK transmission," Electron. Lett., vol. 42, no. 3, pp. 178-179, 2006.

[4] H. C. Hansen Mulvad, M. Galili, L. K. Oxenløwe, H. Hu, A. T. Clausen, J. B. Jensen, C. Peucheret, and P. Jeppesen, "Demonstration of $5.1 \mathrm{Tbit} / \mathrm{s}$ data capacity on a single-wavelength channel," Opt. Exp., vol. 18, no. 2, pp. 1438-1443, Jan. 2010.

[5] P. Martelli, P. Boffi, M. Ferrario, L. Marazzi, P. Parolari, R. Siano, V. Pusino, P. Minzioni, I. Cristiani, C. Langrock, M. M. Fejer, M. Martinelli, and V. Degiorgio, "All-optical wavelength conversion of a 100-Gbit/s polarization-multiplexed signal,” Opt. Exp., vol. 17, no. 20, pp. 17758-17763, Sep. 2009.

[6] M. Galili, J. Xu, H. C. Mulvad, L. K. Oxenløwe, A. T. Clausen, P. Jeppesen, B. Luther-Davies, S. Madden, A. Rode, D.-Y. Choi, M. Pelusi, F. Luan, and B. J. Eggleton, "Breakthrough switching speed with an all-optical chalcogenide glass chip: $640 \mathrm{Gbit} / \mathrm{s}$ demultiplexing," Opt. Exp., vol. 17 no. 4, pp. 2182-2187, Feb. 2009.

[7] T. D. Vo, M. D. Pelusi, J. Schröder, F. Luan, S. J. Madden, D.-Y. Choi, D. A. P. Bulla, B. Luther-Davies, and B. J. Eggleton, "Simultaneous multiimpairment monitoring of $640 \mathrm{Gbit} / \mathrm{s}$ signals using photonic chip based RF spectrum analyzer," Opt. Exp., vol. 18, no. 4, pp. 3938-3945, Feb. 2010.

[8] T. D. Vo, H. Hu, M. Galili, E. Palushani, J. Xu, L. K. Oxenløwe, S. J. Madden, D.-Y. Choi, D. A. P. Bulla, M. D. Pelusi, J. Schroder, B. Luther-Davies, and B. J. Eggleton, "Photonic chip based 1.28 Tbaud transmitter optimization and receiver OTDM demultiplexing," presented at the OFC 2010, San Diego, CA, paper PDPC5.

[9] H. Ji, H Hu, M. Galili, L. K. Oxenløwe, M. Pu, K. Yvind, J. M. Hvam, and P. Jeppesen, "Optical waveform sampling and error-free demultiplexing of $1.28 \mathrm{Tbit} / \mathrm{s}$ serial data in a silicon nanowire," presented at the OFC 2010, San Diego, CA, paper PDPC7.

[10] G. Charlet, J. Renaudier, H. Mardoyan, P. Tran, O. B. Pardo, F. Verluise, M. Achouche, A. Boutin, F. Blache, J.-Y. Dupuy, and S. Bigo, "Transmission of 16.4-bit/s capacity over $2550 \mathrm{~km}$ using PDM QPSK modulation format and coherent receiver," J. Lightw. Technol., vol. 27, no. 3, pp. 153157, Feb. 2009.

[11] X. Q. Jin, R. P. Giddings, E. Hugues-Salas, and J. M. Tang, "Real-time demonstration of 128-QAM-encoded optical OFDM transmission with a $5.25 \mathrm{bit} / \mathrm{s} / \mathrm{Hz}$ spectral efficiency in simple IMDD systems utilizing directly modulated DFB lasers," Opt. Exp., vol. 17, no. 22, pp. 20484-20493, Oct. 2009.

[12] R.-J. Essiambre, G. Kramer, P. J. Winzer, G. J. Foschini, and B. Goebel, "Capacity limits of optical fiber networks," J. Lightw. Technol., vol. 28, no. 4, pp. 662-701, Feb. 2010.

[13] P. J. Winzer, A. H. Gnauck, C. R. Doerr, M. Magarini, and L. L. Buhl, "Spectrally efficient long-haul optical networking using 112-Gbit/s 
polarization-multiplexed 16-QAM," J. Lightw. Technol., vol. 28, no. 4, pp. 547-556, Feb. 2010.

[14] R. S. Tucker, "Switching and energy," presented at the Int. Conf. on Photonics in Switching, Pisa, Italy, Sep. 2009.

[15] F. Parmigiani, S. Asimakis, N. Sugimoto, F. Koizumi, P. Petropoulos, and D. J. Richardson, " $2 \mathrm{R}$ regenerator based on a 2-m-long highly nonlinear bismuth oxide fiber," Opt. Exp., vol. 14, no. 12, pp. 5038-5044, Jun. 2006.

[16] K. K. Chow, K. Kikuchi, T. Nagashima, T. Hasegawa, S. Ohara, and N. Sugimoto, "Four-wave mixing based widely tunable wavelength conversion using 1-m dispersion-shifted bismuth-oxide photonic crystal fiber," Opt. Exp., vol. 15, no. 23, pp. 15418-15423, Nov. 2007.

[17] W. Astar, C.-C. Wei, Y.-J. Chen, J. Chen, and G. M. Carter, "Polarizationinsensitive, $40 \mathrm{Gbit} / \mathrm{s}$ wavelength and RZ-OOK-to-RZ-BPSK modulation format conversion by XPM in a highly nonlinear PCF," Opt. Exp., vol. 16, no. 16, pp. 12039-12049, Aug. 2008.

[18] M. D. Pelusi, F. Luan, E. Magi, M. R. Lamont, D. J. Moss, B. J. Eggleton, J. S. Sanghera, L. B. Shaw, and I. D. Aggarwal, "High bit rate all-optical signal processing in a fiber photonic wire," Opt. Exp., vol. 16, no. 15 , pp. 11506-11512, Jul. 2008.

[19] J. Fatome, C. Fortier, T. N. Nguyen, T. Chartier, F. Smektala, K. Messaad, B. Kibler, S. Pitois, G. Gadret, C. Finot, J. Troles, F. Desevedavy, P. Houizot, G. Renversez, L. Brilland, and N. Traynor, "Linear and nonlinear characterizations of chalcogenide photonic crystal fibers," J. Lightw. Technol., vol. 27, no. 11, pp. 1707-1715, Jun. 2009.

[20] J. Hu, C. R. Menyuk, L. B. Shaw, J. S. Sanghera, and I. D. Aggarwal, "Maximizing the bandwidth of supercontinuum generation in As2Se3 chalcogenide fibers," Opt. Exp., vol. 18, pp. 6722-6739, Mar. 2010.

[21] B. M. N. K. Singhal, "Architectures and algorithm for multicasting in WDM optical mesh networks using opaque and transparent optical crossconnects," in Proc. OFC, Mar. 2001, pp. TuG8-1-TuG8-3.

[22] Y. Zhou and G.-S. Poo, "Optical multicast over wavelength-routed WDM networks: A survey," Opt. Switching Netw., vol. 2, pp. 176-197, 2005.

[23] N. Yan, A. Teixeira, T. Silveira, I. T. Monroy, P. Monteiro, G. Tosi Beleffi, and T. Koonen, "Optical multicasting performance evaluation using multiwavelength conversion by four-wave mixing in DSF at 10/20/40 Gbit/s," in Proc. Photon. Switching, Herakleion, Greece, Oct. 2006, Paper O14.2.

[24] C.-S. Bres, N. Alic, E. Myslivets, and S. Radic, "Scalable multicasting in one-pump parametric amplifier," J. Lightw. Technol., vol. 27, no. 3, pp. 356-363, Feb. 2009.

[25] M. Fok and C. Shu, "Performance investigation of one-to-six wavelength multicasting of ASK/DPSK signal in a highly nonlinear bismuth oxide fiber," J. Lightw. Technol., vol. 27, no. 15, pp. 2953-2957, Aug. 2009.

[26] O. F. Yilmaz, S. R. Nuccio, X. Wu, and A. E. Willner, "40-Gb/s optical packet buffer using conversion/dispersion-based delays," J. Lightw. Technol., vol. 28, pp. 616-623, Feb. 2010.

[27] M. Karasek, J. Kanka, P. Honzatko, J. Vojtech, and J. Radil, "10 Gbs and 40 Gbs multi-wavelength conversion based on nonlinear effects in HNLF," in Proc. ICTON, pp. 155-161, 2006, paper Tu.D1.7.

[28] K. K. Chow and L. Chinlon, "Applications of photonic crystal fibers (PCFs) in nonlinear signal processing," presented at COIN, Jeju, Korea, MoA2-1, 2006.

[29] O. F. Yilmaz, S. Nuccio, Z. Bakhtiari, X. Wu, J. Wang, L. Zhang, and A. Willner, "Wavelength conversion and 9-fold multicasting of a $21.4 \mathrm{Gbit} / \mathrm{s}$ DPSK data channel using supercontinuum generation," presented at Nonlinear Optics, Honolulu, HI, 2009, paper PDPA3.

[30] L. Rau, B. Olsson, and D. J. Blumenthal, "Wavelength multicasting using an ultra high-speed all-optical wavelength converter," presented at Opt. Fiber Commun. Conf., OSA Tech. Dig. Series (Opt. Soc. Amer., 2001), Anaheim, CA, paper WDD52.

[31] L. Xu, N. Chi, K. Yvind, L. Christiansen, L. Oxenløwe, J. Mørk, P. Jeppesen, and J. Hanberg, "7 × 40 Gbit/s base-rate RZ all-optical broadcasting utilizing an electroabsorption modulator," Opt. Exp., vol. 12, pp. 416-420, 2004.

[32] A. A. M. Saleh and J. M. Simmons, "Evolution toward the next-generation core optical network," J. Lightw. Technol., vol. 24, no. 9, pp. 3303-3321, Sep. 2006

[33] E. J. M. Verdurmen, G. D. Khoe, A. M. J. Koonen, and H. de Waardt, "Alloptical data format conversion from WDM to OTDM based on FWM," Microw. Opt. Technol. Lett., vol. 48, pp. 992-994, 2006.

[34] B.-E. Olsson and D. J. Blumenthal, "WDM to OTDM multiplexing using an ultrafast all-optical wavelength converter," IEEE Photon. Technol. Lett., vol. 13, no. 9, pp. 1005-1007, Sep. 2001.
[35] O. F. Yilmaz, L. Christen, X. Wu, S. R. Nuccio, I. Fazal, and A. E. Willner, "Time-slot interchange of $40 \mathrm{Gbits} / \mathrm{s}$ variable length optical packets using conversion-dispersion-based tunable delays," Opt. Lett., vol. 33, pp. 1954-1956, 2008.

[36] C. Schubert, C. Schmidt, S. Ferber, R. Ludwig, and H. G. Weber, "Errorfree all-optical add-drop multiplexing at $160 \mathrm{Gbit} / \mathrm{s}, "$ Electron. Lett., vol. 39, pp. 1074-1076, 2003.

[37] H.-F. Chou, J. E. Bowers, and D. J. Blumenthal, "Compact 160-Gbit/s add-drop multiplexer with a 40-Gbit/s base rate using electroabsorption modulators," IEEE Photon. Technol. Lett., vol. 16, no. 6, pp. 1564-1565, Jun. 2004.

[38] J. Li, B.-E. Olsson, M. Karlsson, and P. A. Andrekson, "OTDM adddrop multiplexer based on XPM-induced wavelength shifting in highly nonlinear fiber," J. Lightw. Technol., vol. 23, no. 9, pp. 2654-2661, Sep. 2005.

[39] H. C. Hansen Mulvad, M. Galili, L. K. Oxenløwe, A. T. Clausen, P. Jeppesen, and L. Grüner-Nielsen, "640 Gbit/s optical time-division add-drop multiplexing in a non-linear optical loop mirror," in Proc. IEEE LEOS Winter Top. Meeting, 2009, pp. 209-210, paper MC4.4.

[40] H. C. H. Mulvad, M. Galili, L. Grüner-Nielsen, L. K. Oxenløwe, A. T. Clausen, and P. Jeppesen, "640 Gbit/s time-division add-drop multiplexing using a non-linear polarisation-rotating fibre loop," in Proc. ECOC 2008, pp. 1-2, paper Tu.3.D.6.

[41] G. Meloni, M. Scaffardi, P. Ghelfi, A. Bogoni, L. Potì, and N. Calabretta, "Ultra-fast all-optical ADD/DROP multiplexer based on 1-meter-long bismuth oxide-based highly-nonlinear fiber," IEEE Photon. Technol. Lett., vol. 17, no. 12, pp. 2661-2663, Dec. 2005.

[42] A. Bogoni, X. Wu, Z. Bakhtiari, S. Nuccio, and A. E. Willner, "640 Gbit/s all-optical add/drop multiplexing based on pump depletion in a PPLN waveguide," presented at Photonics in Switching, Monterey, CA, Jul. 2010, paper PTuB6.

[43] J. Wang, Z. Bakhtiari, Y. Xiao-Li, S. R. Nuccio, O. F. Yilmaz, X. Wu, J. Y. Yang, Y. Yue, I. Fazal, R. Hellwarth, and A. E. Willner, "Phasetransparent optical data exchange of 40-Gbit/s DPSK signals using fourwave-mixing in a highly nonlinear fiber," presented at the OFC 2010, San Diego, CA, paper OMT6.

[44] A. Bogoni, X. Wu, I. Fazal, and A. Willner, "160 Gbit/s time-domain channel extraction/insertion and all-optical logic operations exploiting a single PPLN waveguide," J. Lightw. Technol., vol. 27, no. 23, pp. 42214227, Oct. 2009.

[45] A. Bogoni, X. Wu, I. Fazal, and A. Willner, " $320 \mathrm{Gbit} / \mathrm{s}$ photonic processing based on sum/difference frequency generation and pump depletion in a single PPLN waveguide," Opt. Lett., vol. 34, no. 12, pp. 1825-1827, 2009.

[46] H. S. Hamza and J. S. Deogun, "Wavelength-exchanging cross connects (WEX)-A new class of photonic cross-connect architectures," J. Lightw. Technol., vol. 24, pp. 1101-1111, Mar. 2006.

[47] K. Mori, H. Takara, and M. Saruwatari, "Wavelength interchange with an optical parametric loop mirror," Electron. Lett., vol. 33, no. 6, pp. 520522, Mar. 1997.

[48] A. Chowdhury, S. C. Hagness, and L. McCaughan, "Simultaneous optical wavelength interchange with a two-dimensional second-order nonlinear photonic crystal," Opt. Lett., vol. 25, no. 11, pp. 832-834, Jun. 2000.

[49] K. K. Y. Wong, M. E. Marhic, K. Uesaka, and L. G. Kazovsky, "Demonstration of wavelength exchange in a highly nonlinear fiber," in Proc. ECOC 2001, Amsterdam, The Netherlands, pp. 272-273.

[50] K. Uesaka, K. K. Y. Wong, M. E. Marhic, and L. G. Kazovsky, "Wavelength exchange in a highly nonlinear dispersion-shifted fiber: Theory and experiments," IEEE J. Sel. Top. Quantum Electron., vol. 8, no. 3, pp. 560-568, May/Jun. 2002.

[51] R. W. L. Fung, H. K. Y. Cheung, and K. K. Y. Wong, "Widely tunable wavelength exchange in anomalous-dispersion regime," IEEE Photon. Technol. Lett., vol. 19, no. 22, pp. 1846-1848, Nov. 2007.

[52] M. Shen, X. Xu, T. I. Yuk, and K. K. Y. Wong, "Byte-level parametric wavelength exchange for narrow pulsewidth return-to-zero signals," IEEE Photon. Technol. Lett., vol. 21, no. 21, pp. 1591-1593, Nov. 2009.

[53] J. Wang, Z. Bakhtiari, Y. Xiao-Li, S. R. Nuccio, O. F. Yilmaz, X. Wu, J. Y. Yang, Y. Yue, I. Fazal, R. Hellwarth, and A. E. Willner, "Phasetransparent optical data exchange of 40-Gbit/s DPSK signals using fourwave-mixing in a highly nonlinear fiber," presented at the OFC 2010, San Diego, CA, paper OMT6.

[54] J. Wang, S. R. Nuccio, X. Wu, O. F. Yilmaz, L. Zhang, I. Fazal, J. Y. Yang, Y. Yue, and A. E. Willner, "40-Gbit/s optical data exchange between WDM channels using second-order nonlinearities in PPLN waveguides," presented at the Nonlinear Optics 2009, Honolulu, HI, paper PDPA1. 
[55] J. Wang, S. R. Nuccio, X. Wu, O. F. Yilmaz, L. Zhang, I. Fazal, J. Y. Yang, Y. Yue, and A. E. Willner, " $40 \mathrm{Gbit} / \mathrm{s}$ optical data exchange between wavelength-division-multiplexed channels using a periodically poled lithium niobate waveguide," Opt. Lett., vol. 35, no. 7, pp. 10671069, Apr. 2010.

[56] J. Wang, Z. Bakhtiari, Y. Xiao-Li, O. F. Yilmaz, S. R. Nuccio, X. Wu, H. Huang, J. Y. Yang, Y. Yue, I. Fazal, R. Hellwarth, and A. E. Willner, "Experimental demonstration of data traffic grooming of a single 10Gbit/s TDM tributary channel between two 160-Gbit/s WDM channels," presented at the OFC 2010, San Diego, CA, paper OWF1, 2011.

[57] K. R. Parameswaran, M. Fujimura, M. H. Chou, and M. M. Fejer, "Lowpower all-optical gate based on sum frequency mixing in APE waveguides in PPLN," IEEE Photon. Technol. Lett., vol. 12, no. 6, pp. 654-656, Jun. 2000.

[58] J. Wang, J. Sun, and Q. Sun, "Single-PPLN-based simultaneous halfadder, half-subtracter, and OR logic gate: Proposal and simulation," Opt. Exp., vol. 15, no. 4, pp. 1690-1699, Feb. 2007.

[59] O. F. Yilmaz, S. R. Nuccio, X. Wu, and A. E. Willner, "Tunable N-fold multicasting and pulsewidth of $40 \mathrm{Gbit} / \mathrm{s}$ channels by variable periodic slicing of a supercontinuum," in Proc. CLEO, May 2009, pp. 1-2.

[60] S. Watanabe, "Optical signal processing using nonlinear fibers," J. Opt. Fiber. Commun., vol. 3, pp. 1-24, 2005.

[61] A. Biberman, B. G. Lee, K. Bergman, A. C. Turner-Foster, M. Lipson, M. A. Foster, and A. L. Gaeta, "First demonstration of on-chip wavelength multicasting," in Proc. OFC 2009, San Diego, CA, paper OTuI3.

[62] E. Ciaramella, G. Contestabile, and A. D'Errico, "A novel scheme to detect optical DPSK signals," Photon. Technol. Lett., vol. 16, pp. 2138-2140, 2004.

[63] H. C. H. Mulvad, L. K. Oxenlwe, M. Galili, A. T. Clausen, L. GrunerNielsen, and P. Jeppesen, "1.28 Tbit/s single-polarisation serial OOK optical data generation and demultiplexing," Electron. Lett., vol. 45, pp. 280-281, 2009.

[64] J. P. R. Lacey, M. V. Chan, R. S. Tucker, A. J. Lowery, and M. A. Summerfield, "All-optical WDM to TDM transmultiplexer," Electron. Lett., vol. 30, pp. 1612-1613, 1994.

[65] M. Hayashi, H. Tanaka, K. Ohara, T. Otani, and M. Suzuki, "OTDM transmitter using WDM-TDM conversion with an electroabsorption wavelength converter," IEEE/OSA J. Lightw. Tech., vol. 20, no. 2, pp. 236-242, Feb. 2002

[66] H. Sotobayashi, W. Chujo, and K. Kitayama, "Photonic gateway: TDMto-WDM-to-TDM conversion and reconversion at $40 \mathrm{Gbit} / \mathrm{s}$ (4 channels $\times 10$ Gbits/s)," J. Opt. Soc. Amer. B, vol. 19, pp. 2810-2816, 2002.

[67] X. Wu, A. Bogoni, S. Nuccio, O. F. Yilmaz, M. Scaffardi, and A. E. Willner, "High-speed optical WDM-to-TDM conversion using fiber nonlinearities," IEEE J. Sel. Topics Quantum Electron., vol. 16, no. 5, Sep./Oct. 2010. [Online]. Available: www.ieeexplore.org

[68] J. Yu and P. Jeppesen, "Simultaneous all-optical demultiplexing and regeneration based on self-phase and cross-phase modulation in a dispersion shifted fiber," J. Lightw. Technol., vol. 19, no. 7, pp. 941-949, Jul. 2001

[69] K. Igarashi and K. Kikuchi, "Optical signal processing by phase modulation and subsequent spectral filtering aiming at applications to ultrafast optical communication systems," IEEE J. Sel. Top. Quantum Electron., vol. 14, no. 3, pp. 551-565, May/Jun. 2008.

[70] X. Wu, A.Bogoni, O. F. Yilmaz, S. R. Nuccio, J. Wang, and A. E. Willner, "8-fold 40-320 Gbit/s phase-coherent multiplexing and 320-40 Gbit/s demultiplexing using highly nonlinear fibers," Opt. Lett., vol. 35, no. 11, pp. 1896-1898, Jun. 2010.

[71] J. H. Lee, T. Nagashima, T. Hasegawa, S. Ohara, N. Sugimoto, and K. Kikuchi, "Bismuth-oxide-based nonlinear fiber with a high SBS threshold and its application to four-wave-mixing wavelength conversion using a pure continuous-wave pump," J. Lightw. Technol., vol. 24, no. 1, pp. 2228, Jan. 2006.

[72] S. Liu, K. J. Lee, F. Parmigiani, M. Ibsen, P. Petropoulos, D. Richardson, and K. Gallo, "OTDM to WDM format conversion based on cascaded SHG/DFG in a single PPLN waveguide," presented at OFC 2010, San Diego, CA, paper OWF4.

[73] G.-W. Lu, S. Shinada, H. Furukawa, N. Wada, T. Miyazaki, and H. Ito, "160-Gbit/s tunable all-optical phase-transparent wavelength conversion through cascaded SFG-DFG in a linear-chirped PPLN waveguide," presented at $O F C$ 2010, San Diego, CA, paper OWF3.

[74] A. Bogoni, X. Wu, I. Fazal, and A. E. Willner, "160 Gbit/s time-domain channel extraction/insertion and all-optical logic operations exploiting a single PPLN waveguide," J. Lightw. Technol., vol. 27, no. 19, pp. 42214227, Oct. 2009.
[75] M. V. Drummond, J. D. Reis, R. N. Nogueira, P. P. Monteiro, A. L. Teixeira, S. Shinada, N. Wada, and H. Ito, "Error-free wavelength conversion at $160 \mathrm{Gbit} / \mathrm{s}$ in PPLN waveguide at room temperature," Electron. Lett., vol. 45, no. 22, pp. 1135-1137, 2009

[76] G.-W. Lu, S. Shinada, H. Furukawa, N. Wada, T. Miyazaki, and H. Ito, "160-Gbit/s tunable all-optical phase-transparent wavelength conversion through cascaded SFG-DFG in a linear-chirped PPLN waveguide," presented at the OFC 2010, San Diego, CA.

[77] L. Zhang and A. E. Willner, "Micro-resonators for communication and signal processing applications," in Photonic Microresonator Research and Applications (Springer Series in Optical Sciences). New York: SpringerVerlag, Apr. 2010.

[78] C. Koos, L. Jacome, C. Poulton, J. Leuthold, and W. Freude, "Nonlinear silicon-on-insulator waveguides for all-optical signal processing," Opt. Exp., vol. 15, pp. 5976-5990, 2007.

[79] Q. Lin, O. J. Painter, and G. P. Agrawal, "Nonlinear optical phenomena in silicon waveguides: Modeling and applications," Opt. Exp., vol. 15, pp. 16604-16644, 2007

[80] J. S. Levy, A. Gondarenko, A. C. Turner-Foster, M. A. Foster, A. L. Gaeta, and M. Lipson, "Four-wave mixing in integrated silicon nitride waveguides," presented at the CLEO 2009, Baltimore, MD, CMFF5.

[81] R. Spano, N. Daldosso, M. Cazzanelli, L. Ferraioli, L. Tartara, J. Yu, V. Degiorgio, E. Jordana, J. M. Fedeli, and L. Pavesi, "Bound electronic and free carrier nonlinearities in Silicon nanocrystals at $1550 \mathrm{~nm}$," Opt Exp., vol. 17, pp. 3941-3950, 2009.

[82] Z. Yuan, A. Anopchenko, N. Daldosso, R. Guider, D. Navarro-Urrios, A. Pitanti, R. Spano, and L. Pavesi, "Silicon nanocrystals as an enabling material for silicon photonics," Proc. IEEE, vol. 97, no. 7, pp. 1250-1268, Jul. 2009.

[83] H. Q. Le and S. Di Cecca, "Ultrafast, room-temperature, resonanceenhanced third-order optical susceptibility tensor of an $\mathrm{AlGaAs} / \mathrm{GaAs}$ quantum well," Opt. Lett., vol. 16, pp. 901-903, 1991.

[84] M. D. Pelusi, V. Ta'eed, L. Fu, E. Maqi, M. R. E. Lamont, S. Madden, D.-Y. Choi, D. A. P. Bulla, B. Luther-Davies, and B. J Eggleton, "Applications of highly-nonlinear chalcogenide glass devices tailored for high-speed all-optical signal processing," IEEE J. Sel. Top. Quantum Electron., vol. 14, no. 3, pp. 529-539, May/Jun. 2008.

[85] M. R. E. Lamont, B. Luther-Davies, D. Y. Choi, S. Madden, and B. J. Eggleton, "Supercontinuum generation in dispersion engineered highly nonlinear $(\gamma=10 / \mathrm{W} / \mathrm{m})$ As2 S3) chalcogenide planar waveguide," Opt. Exp., vol. 16, no. 19, pp. 14938-14944, 2008.

[86] V. R. Almeida, Q. F. Xu, C. A. Barrios, and M. Lipson, "Guiding and confining light in void nanostructure," Opt. Lett., vol. 29, pp. 1209-1211, 2004.

[87] L. Zhang, Y. Yue, Y. Xiao-Li, J. Wang, R. G. Beausoleil, and A. E. Willner, "Flat and low dispersion in highly nonlinear slot waveguides," Opt. Exp., vol. 18, no. 12, pp.13187-13193, 2010.

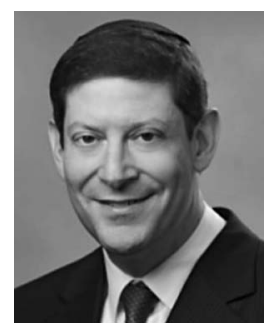

Alan E. Willner (S'87-M'88-SM'93-F'04) received the $\mathrm{Ph} . \mathrm{D}$. degree in electrical engineering from Columbia University, New York, in 1988.

$\mathrm{He}$ was at AT\&T Bell Laboratories and Bellcore. He is currently a Professor at the University of Southern California, Los Angeles. He is the Editor-in-Chief of the OSA Optics Letters. He is the author or coauthor more than 775 papers published in various international journals and conferences, two books, and holds 25 patents.

Prof. Willner's professional activities have included the following: President of the IEEE Lasers and Electro-Optics Society (LEOS), the Editor-in-Chief of the IEEE/OSA JOURNAL OF LIGHTWAVE TECHNOLOGY and the IEEE JOURNAL OF SELECTED TOPICS IN QUANTUM ELECTRONICS, the Co-Chair of the OSA Science and Engineering Council, the General Co-Chair of the Conference on Lasers and Electro-Optics, the Chair of the IEEE TAB Ethics and Conflict Resolution Committee, the Genera Chair of the LEOS Annual Meeting Program, the Program Co-Chair of the OSA Annual Meeting, and Steering and Program Committee Member of the Conference on Optical Fiber Communications. He has received the National Science Foundation (NSF) Presidential Faculty Fellows Award from the White House, the Packard Foundation Fellowship, the NSF National Young Investigator Award, the Fulbright Foundation Senior Scholars Award, the IEEE LEOS Distinguished Traveling Lecturer Award, the University of Southern California University-Wide Award for Excellence in Teaching, and the Eddy Award from Pennwell for the best contributed technical article. He was a Fellow of the Semiconductor Research Corporation and is currently a Fellow of the Optical Society of America (OSA). 


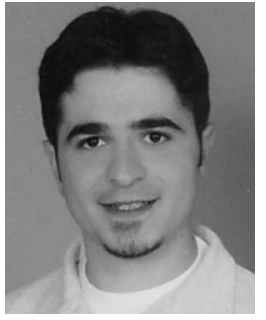

Omer Faruk Yilmaz (S'03) received the B.S. degree from the Middle East Technical University, Turkey, in 2004, and the M.S. degree in electrical engineering from University of California, Riverside, in 2006. $\mathrm{He}$ is currently working toward the Ph.D. degree in electrical engineering at the University of Southern California, Los Angeles.

He is a Reviewer of the journals Optics Express, Optics Letters, and Journal of Lightwave Technology. His current research interests include high-speed optical signal processing, and nonlinear optical-signalprocessing applications on advanced modulation formats.

Mr. Yilmaz is a Student Member of the IEEE Photonics Society and the Optical Society of America. He received the Best Research Paper Award from the Department of Electrical Engineering, University of Southern California along with the Best Teaching Assistant Award in 2009

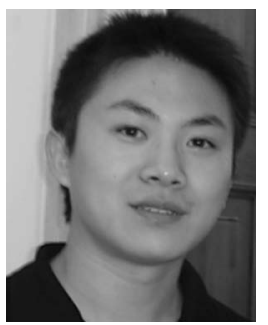

Jian Wang ( $S^{\prime}$ 07) received the B.E. degree in optical information science and technology from the Huazhong University of Science and Technology, Wuhan, China, in 2003, and the Ph.D. degree in physical electronics from the Wuhan National Laboratory for Optoelectronics, Huazhong University of Science and Technology, Wuhan, China, in 2008

He joined the Wuhan National Laboratory for Optoelectronics, Wuhan, China, in 2005. He is currently a Postdoctoral Research Associate in the optical communications laboratory in electrical engineering, University of Southern California, Los Angeles. He is the author or coauthor of more than 50 papers published in international prestigious journals and conferences. He is a Reviewer of the journals Optics Express, Optics Letters, the Journal of the Optical Society of America B, Optics Communications, etc. His research interests include nonlinear optics, optical communications, ultrafast optical signal processing, and integrated photonics.

Dr. Wang is also a Reviewer of the IEEE PHOTONICS TECHNOLOGY LETTERS and the IEEE JOURNAL OF QUANTUM ELECTRONICS.

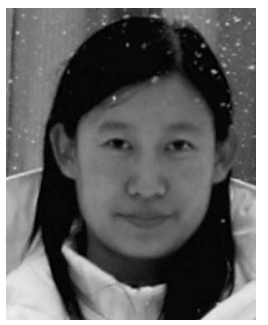

Xiaoxia Wu (S'06) received the B.E. degree from Jilin University, Changchun, China, in 2004, and the M.E degree from the Beijing University of Posts and Telecommunications, Beijing, China, in 2006 She is currently working toward the Ph.D. degree in electrical engineering at the University of Southern California, Los Angeles.

In September 2008, she was a Visiting Student at Consorzio Nazionale Interuniversitario per le Telecomunicazioni, Pisa, Italy. She is the author or coauthor of more than 80 papers published in prestigious international journals and conferences. She is a Reviewer for the journals Optics Letters, Optics Express, and Optical Engineering. Her research interests include high-speed optical signal processing, optical performance monitoring, and advanced optical modulation formats.

Ms. Wu is a Student Member of the IEEE Photonics Society, the Optical Society of America, and the International Society for Optical Engineering. She was one of the recipients of the 2009 IEEE Photonics Society Graduate Student Fellowship Award. She is also a Reviewer for the IEEE PHOTONICS TECHNOLOGY LETTERS and the IEEE PHOTONICS JOURNAL.

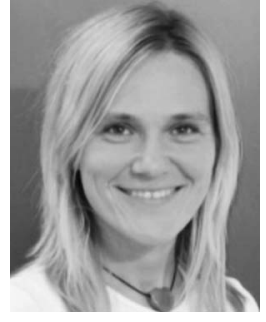

Antonella Bogoni was born in Mantova, Italy in 1972. She received the M.S. degree in electronics engineering, in 1997, and the Ph.D. degree, in 2004 from the University of Parma, Parma, Italy.

From 1998 to 1999, she was a Grantee of Marconi S.p.a. at the University of Parma. From 2000 to 2006, she was a Researcher of Consorzio Nazionale Interuniversitario per le Telecomunicazioni (CNIT) at the University of Parma up to 2001 and then at Photonic Networks National Laboratory, Pisa, Italy. Currently, she is the Head of research of CNIT at the Integrated Research Center for Photonic Networks and Technologies. Since 2006, she has been the CEO of PhoTrix. In 2008 and 2010, she had been a visitor at the University of Southern California, Los Angeles. She has been the Scientific Coordinator for national and international projects and involved in several EU and national projects. She is the coauthor of more than seven books, 60 papers on international journals, 160 contributes for international conferences, and holds 40 international patents. Her research interests include fiber optical transmissions, especially in ultrafast all-optical signal processing and pulsed source generation.

Dr. Bogoni received the Fulbright Advanced Research Scholarship Award for the project "Design and implementation of a 640-Gb/s OTDM system." She was involved with the Revision Committees of international conferences, and she is the Reviewer for international journals and for the European Commission within FP7.

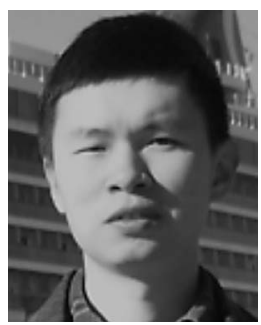

Lin Zhang received the B.S. and M.S. degrees (with honors) from Tsinghua University, Beijing, China, in 2001 and 2004, respectively. He is currently working toward the Ph.D. degree in electrical engineering at the University of Southern California, Los Angeles.

His research interests include microresonator devices and system applications, chip-scale optical interconnection, slow light, and photonic crystal fibers. $\mathrm{He}$ is a Reviewer for Optics Letters and Optics Express. He is the author or coauthor of more than 60 peer-reviewed journal article and conference papers, including five invited papers, one book chapter, and holds two patents.

Mr. Zhang is a Student Member of the IEEE Lasers and Electro-Optics Society (LEOS), the Optical Society of America (OSA), and the International Society of Optical Engineering (SPIE). He is a Reviewer of the IEEE PHOTONICS TECHNOLOGY LETTERS and IEEE/OSA JOURNAL OF LIGHTWAVE TECHNOLOGY. He was cited as one of the 2003 Top-Ten Outstanding Graduate Students at Tsinghua University. He received the Best Research Paper Award from the Department of Electrical Engineering at the University of Southern California and won the Student Poster Competition in the 19th IEEE LEOS Annual Workshop on Interconnects. He is also a recipient of the 2008 HP Innovation Research Award and one of the recipients of the 2008 IEEE LEOS Graduate Student Fellowship.

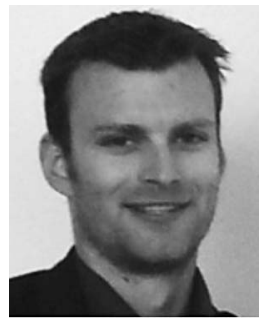

Scott R. Nuccio received the B.S. degree in electrical engineering from the University of California, San Diego, in 2004, and the M.S. degree in electrical engineering, in 2005, from the University of Southern California, Los Angeles, where he is currently working toward the Ph.D. degree in the Optical Communications Laboratory

His research interests include the study and application of advanced modulation formats and of nonlinear optical processes for applications to fiber-optic communication systems. He is also a member of the technical staff at the Aerospace Corporation, El Segundo, CA. 